\title{
NON-ARCHIMEDEAN REACTION-ULTRADIFFUSION EQUATIONS AND COMPLEX HIERARCHIC SYSTEMS
}

\author{
W. A. ZÚÑIGA-GALINDO
}

\begin{abstract}
We initiate the study of non-Archimedean reaction-ultradiffusion equations and their connections with models of complex hierarchic systems. From a mathematical perspective, the equations studied here are the $p$-adic counterpart of the integro-differential models for phase separation introduced by Bates and Chmaj. Our equations are also generalizations of the ultradiffusion equations on trees studied in the 80 s by Ogielski, Stein, Bachas, Huberman, among others, and also generalizations of the master equations of the Avetisov et al. models, which describe certain complex hierarchic systems. From a physical perspective, our equations are gradient flows of nonArchimedean free energy functionals and their solutions describe the macroscopic density profile of a bistable material whose space of states has an ultrametric structure. Some of our results are $p$-adic analogs of some well-known results in the Archimedean setting, however, the mechanism of diffusion is completely different due to the fact that it occurs in an ultrametric space.
\end{abstract}

\section{INTRODUCTION}

In the middle of the 80 s the idea of using ultrametric spaces to describe the states of complex biological systems, which naturally possess a hierarchical structure, emerged in the works of Frauenfelder, Parisi, Stein, among others, see e.g. 18, 20, 28, 31. A central paradigm in physics of complex systems (for instance proteins) asserts that the dynamics of such systems can be modeled as a random walk in the energy landscape of the system, see e.g. [20, 24, and the references therein. In protein physics, it is regarded as one of the most profound ideas put forward to explain the nature of distinctive life attributes. Typically these landscapes have a huge number of local minima. It is clear that a description of the dynamics on such landscapes requires an adequate approximation. The interbasin kinetics method offers an acceptable solution to this problem. The idea is to study the kinetics generated by transitions between groups of states (basins). In this framework, the minimal basins correspond to local minima of energy, and the large basins (superbasins or union of basins) have a hierarchical structure. By using this approach an energy landscape is approximated by an ultrametric space (called a disconnectivity graph) and a function on this space describing the distribution of the activation barriers, see e.g. [13]. An ultrametric space $(M, d)$ is a metric space $M$ with a distance satisfying $d(A, B) \leq \max \{d(A, C), d(B, C)\}$ for any three points $A, B, C$ in $M$. Mezard, Parisi, Sourlas and Virasoro discovered, in the context of the mean-field theory of spin glasses, that the space of states of such systems

2000 Mathematics Subject Classification. Primary 80A22, 45K05; Secondary 46S10.

Key words and phrases. Phase separation, energy landscapes, free-energy functionals, reactiondiffusion equations, ultrametricity, ultrametric spaces, complex systems, $p$-adic analysis.

The author was partially supported by Conacyt Grant No. 250845. 
has an ultrametric structure, see e.g. 28, [31. The rooted trees have a natural structure of ultrametric space, and the disconnectivity graph above mentioned is an example of a such space.

After that, a model of hierarchical dynamics is constructed, and by using the postulates of the interbasin kinetics, one gets that the transitions between basins are described by the following equations:

$$
\frac{\partial u(i, t)}{\partial t}=\sum_{j \neq i} T(j, i) v(j) u(j, t)-\sum_{j \neq i} T(i, j) v(i) u(i, t), i=1, \ldots, N,
$$

where the indices $i, j$ number the states of the system (which correspond to local minima of energy), $T(i, j) \geq 0$ is the probability per unit time (or transition rate) of a transition from $i$ to $j$, and the $v(j)>0$ are the basin volumes. At this point it is relevant to mention that equations of type (1.1) are a generalization of the ultradiffusion equations on trees studied intensively in the $80 \mathrm{~s}$, see e.g. 10 and the references therein, and that these equations appeared in models of protein folding see e.g. 39 .

Along this article $p$ will denote a fixed prime number. The field of $p$-adic numbers $\mathbb{Q}_{p}$ is defined as the completion of the field of rational numbers $\mathbb{Q}$ with respect to the $p$-adic norm $|\cdot|_{p}$. See Section 2 for the essential ideas about $p$-adic analysis. Here, we just mention that in the $p$-adic norm, the integers highly divisible by $p$ are small. For instance, if $p=2$, then $\left|2^{k}\right|_{2}=2^{-k}$, for a positive integer $k$, while for $p=3,\left|2^{k}\right|_{3}=1$. A such norm satisfies $|x+y|_{p} \leq \max \left\{|x|_{p},|y|_{p}\right\}$, and the metric space $\left(\mathbb{Q}_{p},|\cdot|_{p}\right)$ is a complete ultrametric space. This space has a natural hierarchical structure, which is very useful in physical models involving hierarchies. As a topological space $\mathbb{Q}_{p}$ is homeomorphic to a Cantor-like subset of the real line, i.e. $\mathbb{Q}_{p}$ is a fractal. The $p$-adic norm can be extended to $\mathbb{Q}_{p}^{n}$ by taking for $x=\left(x_{1}, \cdots, x_{n}\right),\|x\|_{p}:=\max _{i}\left|x_{i}\right|_{p}$.

Around 2000, Avetisov et al. discovered, among several things, that under suitable physical and mathematical hypotheses, the ultradiffusion equations on trees studied by Ogielski, Stain, Bachas and Huberman, among several others, see e.g. [10, have a 'continuous $p$-adic limit'. We explain briefly these ideas following [30] and $\left[9\right.$. The states of system are labeled by the numbers $0,1, \ldots, 2^{n-1}-1$, for some $n$. By representing each number in base 2 , we get a set of $2^{n}$ binary vectors of lenght $n$. This set forms a rooted tree with $n+1$ levels and $2^{n}$ branches (states). An ultrametric distance $d$ between two branches is given by the number of levels that it is necessary to descend up to the branches merge. Now, a stochastic dynamical system on the rooted tree is introduced by using a random walk (on the top level) to model the transition between states. We denote by $P_{i}(t)$ the probability of occupying the state $i$ at the time $t$, and set $\boldsymbol{P}(t)=\left[P_{0}(t), \ldots, P_{2^{n}-1}(t)\right]^{T}$. We now assume that the probability per unit of time of jumping from state $i$ into state $j$ is a function of the ultrametric distance, in this way we construct a matrix $Q=[f(d(i, j))]$, the Parisi matrix of the system, and the dynamics of the system is controlled by the master equation

$$
\frac{d \boldsymbol{P}(t)}{d t}=Q \boldsymbol{P}(t) .
$$

See 30 for further details. On the other hand, in the models of spontaneous breaking of the replica symmetry are used for the investigation of spin glasses, a replica matric $Q=\left[Q_{a b}\right]$ of size $n \times n$ occurs. This matrix is constructed as follows: 
consider the set of integers $m_{i}$, with $i \in\{1, \ldots, N\}$, where $\frac{m_{i}}{m_{i-1}}$ are integers for $i>1$ and $\frac{n}{m_{i}}$ are also integers. The entries of the replica matrix are defined as follows:

$$
Q_{a a}=0, \text { and for } a \neq b, Q_{a b}=q_{i} \text {, with }\left[\frac{a}{m_{i}}\right] \neq\left[\frac{b}{m_{i}}\right] \text { and }\left[\frac{a}{m_{i+1}}\right]=\left[\frac{b}{m_{i+1}}\right] \text {, }
$$

here $[\cdot]$ denotes the integer part function. To obtain a $p$-adic parametrization of the replica matrix (the Parisi matrix), we use the set $\left\{1, \ldots, p^{N}\right\}$ (instead of $\{1, \ldots, N\}$ ) and the mapping $l:\left\{1, \ldots, p^{N}\right\} \rightarrow p^{-N} \mathbb{Z} / \mathbb{Z}$ defined as $l^{-1}\left(\sum_{j=1}^{N} x_{j} p^{-j}\right)=1+$ $p^{-1} \sum_{j=1}^{N} x_{j} p^{j}$, where $x_{j}$ are $p$-adic digits. In [9], Avetisov et al. established that the replica matrix can be parametrized as $Q_{a b}=f\left(|l(a)-l(b)|_{p}\right)$ where $f$ is a function such that $f\left(p^{i}\right)=q_{i}$ and $f(0)=0$, and that in 'the limit $N$ tends to infinity,' master equation (1.2) becomes the $p$-adic heat equation:

$$
\frac{\partial u(x, t)}{\partial t}+D_{x}^{\alpha} u(x, t)=0, \quad x \in \mathbb{Q}_{p}, t \geq 0
$$

where $D_{x}^{\alpha}$ is the Vladimirov operator, and the matrix $\left[Q_{a b}\right]$ represents this operator in a convenient finite dimensional space, i.e. $\left[Q_{a b}\right]$ is a discretization of the Vladimirov operator. For further details, the reader may consult [9], [23], 38]. The terminology ' $p$-adic heat equation' means that a Markov process is attached to the fundamental solution of (1.3), consequently, we can consider (1.3) as a $p$-adic analogue of the classical heat equation. For an in-depth discussion of this analogy, the reader may consult [23, 38] and the references therein. On the other hand, many different ultrametrics can be defined on a rooted tree, at first sight, the above limit process works only for ultrametrics of the form $f \circ|\cdot|_{p}$, however, this matter has not been investigated yet.

We now come back to equation (1.1) to explain how the above discussed ideas fit into it. We rewrite (1.1) as follows:

$$
\frac{\partial}{\partial t}\left[\begin{array}{c}
u(1, t) \\
\vdots \\
u(j, t) \\
\vdots \\
u(N, t)
\end{array}\right]=\left[W(j, i)-\delta_{i i} W(i, i)\right]_{N \times N}\left[\begin{array}{c}
u(1, t) \\
\vdots \\
u(j, t) \\
\vdots \\
u(N, t)
\end{array}\right]
$$

where $W(j, i)=T(j, i) v(j)$, for $j \neq i$, and $W(i, i)=v(i) \sum_{j \neq i} T(i, j)$. We assume that the space of states $\{1, \ldots, N\}$ have a hierarchical structure, then $\left[W(j, i)-\delta_{i i} W(i, i)\right]$ is a Parisi-type matrix. These matrices appear naturally in models of complex systems such as spin glasses, see e.g. [10], [28, [30, [31, [23] and the references therein. Under mild hypotheses, these Parisi matrices can be parametrized by $p$-adic numbers, and then the master equation (1.4) becomes a discretization of $p$-adic ultradiffusion equation of type (1.5). Consequently, it is completely natural to propose that in the limit when $N$ tends to infinity the master equation (1.4) becomes a $p$-adic ultradiffusion equation, see e.g. [23, Chapters 4 ,8], [38, Chapter 2,3] and the references therein. 
The ' $p$-adic limit' of master equations (1.4) have the form:

$$
\frac{\partial u(x, t)}{\partial t}=\int_{\mathbb{Q}_{p}^{n}} J\left(\|x-y\|_{p}\right)[u(y, t)-u(x, t)] d^{n} y,
$$

$x \in \mathbb{Q}_{p}^{n}, t \geq 0$. The function $u(x, t): \mathbb{Q}_{p}^{n} \times \mathbb{R}_{+} \rightarrow \mathbb{R}_{+}$is a probability density distribution, so that $\int_{B} u(x, t) d^{n} x$ is the probability of finding the system in a domain $B \subset \mathbb{Q}_{p}^{n}$ at the instant $t$. The function $J\left(\|x-y\|_{p}\right): \mathbb{Q}_{p}^{n} \times \mathbb{Q}_{p}^{n} \rightarrow \mathbb{R}_{+}$is the probability of the transition per unit of time (or transition rate) from state $y$ to state $x$. It is known that for many $J_{\mathrm{s}}$, equations of type (1.5) are ultradiffusion equations i.e. they are $p$-adic counterparts of the classical heat equations. For instance, $J\left(\|x\|_{p}\right)=\|x\|_{p}^{\gamma} e^{-\|x\|_{p}}$, with $\gamma>-n$, corresponds to an exponential landscape in the sense of [6], in this case, the fundamental solution of (1.5) is the transition density of a bounded right-continuous Markov process without second kind discontinuities, see 34, 14 and the references therein. As a consequence of the work of many people, among them, Vlamimirov, Volovich, Zelenov, Avetisov, Kozyrev, Kochubei, Khrennikov, Albeverio, and Zúñiga-Galindo, we have now a good theory of $p$-adic 'linear' reaction-diffusion equations which has emerged motivated by connections between $p$-adic analysis and models of complex systems. For further details the reader may consult [38, [23], see also [34, as well as the classics, [36], 22].

In our opinion, the novelty and relevance of the 'idealistic models' of Avetisov et al. come from two facts: first, they codify, in a mathematical language, the central physical paradigm asserting that the dynamics of (many) complex systems can be described as a random walk on an ultrametric space; second, these models give a description of the characteristic types of relaxation of complex systems. The original models of Avetisov et al. were formulated in dimension one, more precisely, these models were constructed by using 'exactly one' cross section of an energy landscape. In [20, p. 98 and figures 11.3 and 11.4] Frauenfelder et al. have pointed out that using 'one' cross section of an energy landscape of a complex systems to describe its dynamics is misleading, because it appears that the transition from an initial state to a final state must follow a unique pathway, and entropy does not play a role. By considering several trees and by using the above mentioned limit process, one gets $n$-dimensional $p$-adic master equations of type (1.5). The Frauenfelder et al. observation and the Avetisov et al. work provide a strongly motivation for developing a general theory of $n$-dimensional $p$-adic reaction-ultradiffusion equations.

From the perspectives of mathematics and physics, a natural step in the investigation of equations of types (1.4)-(1.5) is to introduce a non-linear reaction term. This article aims to initiate the theory of $n$-dimensional $p$-adic reactionultradiffusion equations, and their connections with models of complex hierarchic systems. The terminology 'reaction-diffusion equations' has been used in connection with the models of Avetisov et al., see e.g. [15, to mean (linear) parabolic-type equations with variable coefficients. A general theory for this type of equations is given in [38, see also [23], 22]. Here 'reaction-diffusion equations' means nonlinear equations, such as is commonly used in the Euclidean case, see e.g. [19], 21], 32. We use the term ultradiffusion instead of diffusion, due to fact that in classical probability the term diffusion is used only in connection with stochastic processes with continuous paths, and in the $p$-adic setting, the paths cannot be continuous. 
We study equations of the type

$$
\frac{\partial u(x, t)}{\partial t}=\int_{\mathbb{Q}_{p}^{n}} J\left(\|x-y\|_{p}\right)[u(y, t)-u(x, t)] d^{n} y-\lambda f(u(x, t)),
$$

where $J\left(\|x\|_{p}\right) \geq 0, \int_{\mathbb{Q}_{p}^{n}} J\left(\|x\|_{p}\right) d^{n} x=1, \lambda>0$ sufficiently large and $f$ is (for instance) a polynomial having roots in $-1,0,1$. Formally, equation (1.6) is the $L^{2}$ gradient flow of the following non-Archimedean Helmholtz free-energy functional:

$$
E[\varphi]=\frac{1}{4} \int_{\mathbb{Q}_{p}^{n}} \int_{\mathbb{Q}_{p}^{n}} J\left(\|x-y\|_{p}\right)\{\varphi(x)-\varphi(y)\}^{2} d^{n} x d^{n} y+\lambda \int_{\mathbb{Q}_{p}^{n}} W(\varphi(x)) d^{n} x,
$$

where $\varphi$ is a function taking values in the interval $[-1,1]$ and $W$ is a double-well potential.

Equations of the form (1.6) can be well-approximated in finite dimensional real spaces by ODE's. In a suitable basis, where the unknown function is identified with the column vector $[u(i, t)]_{i \in G_{N}^{n}}$, these equations have the form

$$
\frac{\partial}{\partial t}[u(\boldsymbol{i}, t)]_{i \in G_{N}^{n}}=-A^{(N)}[u(\boldsymbol{i}, t)]_{\boldsymbol{i} \in G_{N}^{n}}-\lambda[f(u(\boldsymbol{i}, t))]_{\boldsymbol{i} \in G_{N}^{n}},
$$

where $A^{(N)}$ is the matrix representation of a linear operator that approximates, in a suitable finite dimensional vector space, the integral operator involving the function $J$ in the right-side of (1.6). Equation (1.8) is $L^{2}$-gradient flow of a 'finite' Helmholtz energy functional, i.e. a functional defined on the space $G_{N}^{n}$. In Section 5 , we present some results about the convergence of finite Helmholtz functionals when $N$ tends to infinity. Equations of type (1.8) are generalizations of ultradiffusion equations on trees considered in [10]. The set $G_{N}^{n}$ is a finite ultrametric space, this class of spaces contains as particular case the finite rooted trees.

This article is dedicated to study the interplay between all the above mentioned objects and their physical significance. We determine the spaces and conditions for which the Cauchy problems for equations (1.6)-(1.8) are well-posed, see Theorems [5. 6. We show that equations (1.6)-(1.8) have stationary solutions with 'arbitrary interfaces', this means, in the case of equation (1.6), the following. Given a ball $B_{N_{0}}^{n}\left(x_{0}\right)$ of radius $p^{N_{0}}$ centered at $x_{0}, \mathbb{Q}_{p}^{n}$ can be divided into three disjoint sets $M, B_{N_{0}}^{n}\left(x_{0}\right) \backslash M$, and $\mathbb{Q}_{p}^{n} \backslash B_{N_{0}}^{n}\left(x_{0}\right)$. Equation (1.6) admits a stationary solution $\widetilde{u}(x)$ satisfying $\alpha^{+} \leq \widetilde{u}(x) \leq 1$ for $x \in M,-1 \leq \widetilde{u}(x) \leq \alpha^{-}$for $x \in B_{N_{0}}^{n}\left(x_{0}\right) \backslash M$, and $\lim _{\|x\|_{p} \rightarrow \infty} \widetilde{u}(x)=0$, for some suitable constants $\alpha^{+}, \alpha^{-}$, see Theorems 2 , 3 .

We also show that the solution of Cauchy problem attached to (1.8) converges to the solution of the Cauchy problem attached to (1.6), when $N$ tends to infinity, in the case in which the initial condition for equation (1.8) is a continuous function taking values in the interval $[-1,1]$, see Theorem 7 , Roughly speaking, equation (1.6) is the ' $p$-adic continuous limit' of the system of equations (1.8), when $N$ tends to infinity. The matrix $A^{(N)}$ in equation (1.8) is the $Q$-matrix of a finite homogeneous Markov chain with state space $G_{N}^{n}$, and equation (1.8) with $f=0$ is the Kolmogorov backward equation attached to this Markov chain, see Theorem 1

From a physical perspective equations (1.6)-(1.8) model phase separation of bistable materials whose space of states have an ultrametric structure. Our models are the $p$-adic counterparts of the integro-differential models for phase separation 
due to Bates and Chmaj, see [11-12, and [2]-33. The function $u(x, t)$, respectively $[u(i, t)]_{i \in G_{N}^{n}}$, the order parameter, represents the macroscopic density profile of a material, which has two equilibrium states $u(x, t) \equiv-1, u(x, t) \equiv 1$, and $-1<u(x, t)<1$ represents the 'interface', and equations (1.6)-(1.8) model a transition between the equilibrium phases. Theorems 2 , 3 show that our models of bistable systems can develop arbitrary stable interfaces.

In the proofs of our results we have used freely techniques of abstract evolution equations, for instance [16, 29], and adapted techniques and ideas of the classical reaction-diffusion equations, for instance [21, 32]. However, the non-Archimedean theory is far from being a straightforward consequence of the classical theory of reaction-diffusion equations. For instance, the existence of traveling waves, that usually emerge in the transformation between the pure phases, is an open problem in the non-Archimedean case, since the classical ideas cannot be applied directly. On the other hand, the non-Archimedean comparison theorem needed here, see Theorem 4 and Corollary 2, requires the condition that the "volume of the system be sufficiently large'.

In a recent book by Dellacherie, Martinez and San Martin, see [17, the authors present a theory of 'ultrametric matrices' and their connections with Markov chains. As far as we understand, these ultrametric matrices are generalizations of the inverses of Parisi matrices. In particular, the results of this book imply that our Theorem 1 is valid for more general type of matrices. It is interesting to mention that Khrennikov and Kozyrev developed a very general theory of Parisi-type matrices, see [25]-27].

Finally, our Theorem 7 allows us to produce numerical simulations of the behavior of the bistable systems whose states are described by the solutions of our $p$-adic reaction-ultradiffusion equations. We prefer focus our article on mathematical aspects, however, our equations include as a particular case the reaction-diffusion equations on graphs studied by Ueyama and Hosoe in [35. The numerical simulations presented in this article show that our $p$-adic reaction-ultradiffusion equations develop stable patterns such as occurs in the Archimedean case. Here, it is important to mention that 'essentially' there is no available literature on numerical methods for nonlinear $p$-adic reaction-ultradiffusion equations. For some special equations, numerical solutions can be obtained using $p$-adic wavelets, see [23], 24], and the references therein. In our opinion, this technique is not applicable to the type of equations considered here. There are well-known mathematical techniques for the discretization abstract nonlinear evolution equations, we use some of them here, but the challenge is the 'visualization of the data.' Then, the numerical study of the stable patterns of the equations introduced here, and the comparison with the stable patterns corresponding to the classical reaction-diffusion equations is an open problem.

\section{2. $p$-Adic Analysis: Essential Ideas}

2.1. The field of $p$-adic numbers. Along this article $p$ will denote a prime number. The field of $p$-adic numbers $\mathbb{Q}_{p}$ is defined as the completion of the field of rational numbers $\mathbb{Q}$ with respect to the $p$-adic norm $|\cdot|_{p}$, which is defined as

$$
|x|_{p}=\left\{\begin{array}{lll}
0 & \text { if } & x=0 \\
p^{-\gamma} & \text { if } & x=p^{\gamma} \frac{a}{b},
\end{array}\right.
$$


where $a$ and $b$ are integers coprime with $p$. The integer $\gamma:=\operatorname{ord}(x)$, with $\operatorname{ord}(0):=$ $+\infty$, is called the $p$-adic order of $x$. We extend the $p$-adic norm to $\mathbb{Q}_{p}^{n}$ by taking

$$
\|x\|_{p}:=\max _{1 \leq i \leq n}\left|x_{i}\right|_{p}, \quad \text { for } x=\left(x_{1}, \ldots, x_{n}\right) \in \mathbb{Q}_{p}^{n} .
$$

We define $\operatorname{ord}(x)=\min _{1 \leq i \leq n}\left\{\operatorname{ord}\left(x_{i}\right)\right\}$, then $\|x\|_{p}=p^{-\operatorname{ord}(x)}$. The metric space $\left(\mathbb{Q}_{p}^{n},\|\cdot\|_{p}\right)$ is a complete ultrametric space. As a topological space $\mathbb{Q}_{p}$ is homeomorphic to a Cantor-like subset of the real line, see e.g. 1], 36.

Any $p$-adic number $x \neq 0$ has a unique expansion of the form

$$
x=p^{\operatorname{ord}(x)} \sum_{j=0}^{\infty} x_{i} p^{j},
$$

where $x_{j} \in\{0,1,2, \ldots, p-1\}$ and $x_{0} \neq 0$. In addition, any $p$-adic number $x \neq 0$ can be represented uniquely as $x=p^{o r d(x)} a c(x)$ where $a c(x)=\sum_{j=0}^{\infty} x_{i} p^{j}, x_{0} \neq 0$, is called the angular component of $x$. Notice that $|a c(x)|_{p}=1$.

2.2. Topology of $\mathbb{Q}_{p}^{n}$. For $r \in \mathbb{Z}$, denote by $B_{r}^{n}(a)=\left\{x \in \mathbb{Q}_{p}^{n} ;\|x-a\|_{p} \leq p^{r}\right\}$ the ball of radius $p^{r}$ with center at $a=\left(a_{1}, \ldots, a_{n}\right) \in \mathbb{Q}_{p}^{n}$, and take $B_{r}^{n}(0):=B_{r}^{n}$. Note that $B_{r}^{n}(a)=B_{r}\left(a_{1}\right) \times \cdots \times B_{r}\left(a_{n}\right)$, where $B_{r}^{1}\left(a_{i}\right):=B_{r}\left(a_{i}\right)=\left\{x \in \mathbb{Q}_{p} ;\left|x_{i}-a_{i}\right|_{p} \leq\right.$ $\left.p^{r}\right\}$ is the one-dimensional ball of radius $p^{r}$ with center at $a_{i} \in \mathbb{Q}_{p}$. The ball $B_{0}^{n}$ equals the product of $n$ copies of $B_{0}=\mathbb{Z}_{p}$, the ring of $p$-adic integers. We also denote by $S_{r}^{n}(a)=\left\{x \in \mathbb{Q}_{p}^{n} ;\|x-a\|_{p}=p^{r}\right\}$ the sphere of radius $p^{r}$ with center at $a=\left(a_{1}, \ldots, a_{n}\right) \in \mathbb{Q}_{p}^{n}$, and take $S_{r}^{n}(0):=S_{r}^{n}$. We notice that $S_{0}^{1}=\mathbb{Z}_{p}^{\times}$(the group of units of $\mathbb{Z}_{p}$ ), but $\left(\mathbb{Z}_{p}^{\times}\right)^{n} \subsetneq S_{0}^{n}$. The balls and spheres are both open and closed subsets in $\mathbb{Q}_{p}^{n}$. In addition, two balls in $\mathbb{Q}_{p}^{n}$ are either disjoint or one is contained in the other.

As a topological space $\left(\mathbb{Q}_{p}^{n},\|\cdot\|_{p}\right)$ is totally disconnected, i.e. the only connected subsets of $\mathbb{Q}_{p}^{n}$ are the empty set and the points. A subset of $\mathbb{Q}_{p}^{n}$ is compact if and only if it is closed and bounded in $\mathbb{Q}_{p}^{n}$, see e.g. [36, Section 1.3], or [1, Section 1.8]. The balls and spheres are compact subsets. Thus $\left(\mathbb{Q}_{p}^{n},\|\cdot\|_{p}\right)$ is a locally compact topological space.

We will use $\Omega\left(p^{-r}\|x-a\|_{p}\right)$ to denote the characteristic function of the ball $B_{r}^{n}(a)$. For more general sets, we will use the notation $1_{A}$ for the characteristic function of a set $A$.

2.3. The Bruhat-Schwartz space. A real-valued function $\varphi$ defined on $\mathbb{Q}_{p}^{n}$ is called locally constant if for any $x \in \mathbb{Q}_{p}^{n}$ there exists an integer $l(x) \in \mathbb{Z}$ such that

$$
\varphi\left(x+x^{\prime}\right)=\varphi(x) \text { for } x^{\prime} \in B_{l(x)}^{n} .
$$

A function $\varphi: \mathbb{Q}_{p}^{n} \rightarrow \mathbb{R}$ is called a Bruhat-Schwartz function (or a test function) if it is locally constant with compact support. Any test function can be represented as a linear combination, with real coefficients, of characteristic functions of balls. The $\mathbb{R}$-vector space of Bruhat-Schwartz functions is denoted by $\mathcal{D}\left(\mathbb{Q}_{p}^{n}\right)$. For $\varphi \in \mathcal{D}\left(\mathbb{Q}_{p}^{n}\right)$, the largest number $l=l(\varphi)$ satisfying (2.1) is called the exponent of local constancy (or the parameter of constancy) of $\varphi$. Let denote by $\mathcal{D}_{N}^{l}\left(\mathbb{Q}_{p}^{n}\right)$ (or simply $\mathcal{D}_{N}^{l}$ ), the finite dimensional subspace of all real-valued test functions having supports in $B_{N}^{n}$ and with parameters of constancy $\geq l$. Then $\mathcal{D}_{N}^{l} \subset \mathcal{D}_{N^{\prime}}^{l^{\prime}}$ if $N^{\prime} \geq N$ and $l \geq l^{\prime}$. 
If $U$ is an open subset of $\mathbb{Q}_{p}^{n}, \mathcal{D}(U)$ denotes the space of test functions with supports contained in $U$, then $\mathcal{D}(U)$ is dense in

$$
L^{\rho}(U)=\left\{\varphi: U \rightarrow \mathbb{R} ;\left(\int_{\mathbb{Q}_{p}^{n}}|\varphi(x)|^{\rho} d^{n} x\right)^{\frac{1}{\rho}}<\infty\right\},
$$

where $d^{n} x$ is the Haar measure on $\mathbb{Q}_{p}^{n}$ normalized by the condition $\operatorname{vol}\left(B_{0}^{n}\right)=1$, for $1 \leq \rho<\infty$, see e.g. [1, Section 4.3]. In the case $U=\mathbb{Q}_{p}^{n}$, we will use the notation $L^{\rho}$ instead of $L^{\rho}\left(\mathbb{Q}_{p}^{n}\right)$. For an in depth discussion about $p$-adic analysis the reader may consult [1, 22, 33, 36.

\section{Some Functional Spaces and Operators}

We define $X_{\infty}\left(\mathbb{Q}_{p}^{n}\right):=X_{\infty}=\overline{\left(\mathcal{D}\left(\mathbb{Q}_{p}^{n}\right),\|\cdot\|_{\infty}\right)}$, where $\|\phi\|_{\infty}=\sup _{x \in \mathbb{Q}_{p}^{n}}|\phi(x)|$ and the bar means the completion with respect the metric induced by $\|\cdot\|_{\infty}$. We also use $\|\cdot\|_{\infty}$ to denote the extension of $\|\cdot\|_{\infty}$ to $X_{\infty}$. Notice that all the functions in $X_{\infty}$ are continuous and that

$$
X_{\infty} \subset C_{0}:=\left(\left\{f: \mathbb{Q}_{p}^{n} \rightarrow \mathbb{R} ; f \text { continuous with } \lim _{\|x\|_{p} \rightarrow \infty} f(x)=0\right\},\|\cdot\|_{\infty}\right) .
$$

On the other hand, since $\mathcal{D}\left(\mathbb{Q}_{p}^{n}\right)$ is dense in $C_{0}$, cf. [33, Chap. II, Proposition 1.3], we conclude that $X_{\infty}=C_{0}$. In a more general case, if $K$ is an open subset of $\mathbb{Q}_{p}^{n}$, we define $X_{\infty}(K)=\overline{\left(\mathcal{D}(K),\|\cdot\|_{\infty}\right)}$.

We set

$$
X_{N}:=\left(\mathcal{D}_{N}^{-N}\left(\mathbb{Q}_{p}^{n}\right),\|\cdot\|_{\infty}\right) \text { for } N \geq 1 .
$$

Any $\varphi \in X_{N}$ has support in $B_{N}^{n}=\left(p^{-N} \mathbb{Z}_{p}\right)^{n}$, and $\varphi$ satisfies (2.1) for $x^{\prime} \in B_{-N}^{n}=$ $\left(p^{N} \mathbb{Z}_{p}\right)^{n}$, in addition, $B_{+N}^{n}$ are additive subgroups and $G_{N}^{n}:=B_{N}^{n} / B_{-N}^{n}$ is a finite group with $\# G_{N}^{n}:=p^{2 N n}$ elements. Any element $\boldsymbol{i}=\left(\boldsymbol{i}_{1}, \ldots, \boldsymbol{i}_{n}\right)$ of $G_{N}^{n}$ can be represented as

$$
\boldsymbol{i}_{j}=a_{-N}^{j} p^{-N}+a_{-N+1}^{j} p^{-N+1}+\ldots+a_{0}^{j}+a_{1}^{j} p+\ldots+a_{N-1}^{j} p^{N-1}
$$

for $j=1, \ldots, n$, with $a_{k}^{j} \in\{0,1, \ldots, p-1\}$. From now on, we fix a set of representatives in $\mathbb{Q}_{p}^{n}$ for $G_{N}^{n}$ of the form (3.1). We denote by $\Omega\left(p^{M}\left\|x-x_{0}\right\|_{p}\right)$, the characteristic function of the ball $x_{0}+\left(p^{M} \mathbb{Z}_{p}\right)^{n}$. We notice that any non-zero function $\varphi$ in $X_{N}$ has an index of local constancy $l_{\varphi} \in\{-N,-N+1, \ldots, 0,1, \ldots, N\}$, and that $B_{l_{\varphi}}^{n}$ can be covered by a finite disjoint union of balls of the form $B_{-N}^{n}(\boldsymbol{j})$, with $\boldsymbol{j} \in G_{N}^{n}$, then $\left\{\Omega\left(p^{N}\|x-\boldsymbol{i}\|_{p}\right)\right\}_{\boldsymbol{i} \in G_{N}^{n}}$ is a basis of $\mathcal{D}_{N}^{-N}$, see also e.g. [1,

Lemma 4.3.1]. We notice now that if $\varphi(x)=\sum_{\boldsymbol{i} \in G_{N}^{n}} \varphi(\boldsymbol{i}) \Omega\left(p^{N}\|x-\boldsymbol{i}\|_{p}\right)$, with $\varphi(\boldsymbol{i}) \in \mathbb{R}$, then $\|\varphi\|_{\infty}=\max _{\boldsymbol{i}}|\varphi(\boldsymbol{i})|$. Hence $X_{N}$ is isomorphic as a Banach space to $\left(\mathbb{R}^{\# G_{N}^{n}},\|\cdot\|_{\mathbb{R}}\right)$, where $\left\|\left(t_{1}, \ldots, t_{\left.\# G_{N}^{n}\right)}\right)\right\|_{\mathbb{R}}=\max _{1 \leq j \leq \# G_{N}^{n}}\left|t_{j}\right|$.

We now define for $N \geq 1, P_{N}: X_{\infty} \rightarrow X_{N}$ as

$$
P_{N} \varphi(x)=\sum_{i \in G_{N}^{n}} \varphi(i) \Omega\left(p^{N}\|x-i\|_{p}\right) .
$$

Therefore $P_{N}$ is a linear bounded operator, indeed, $\left\|P_{N}\right\| \leq 1$.

Lemma 1. $\lim _{N \rightarrow \infty}\left\|\varphi-P_{N} \varphi\right\|_{\infty}=0$ for any $\varphi \in X_{\infty}$. 
Proof. By using the fact that $\mathcal{D}\left(\mathbb{Q}_{p}^{n}\right)$ is dense in $X_{\infty}$, given any $\epsilon$ sufficiently small, there exists $\widetilde{\varphi} \in \mathcal{D}_{M}^{l}\left(\mathbb{Q}_{p}^{n}\right)$, with $l, M$ integers depending on $\epsilon$, such that $\|\varphi-\widetilde{\varphi}\|_{\infty}<$ $\epsilon$. We may assume without loss of generality that $M \geq 1$ since $\mathcal{D}_{M}^{l} \subset \mathcal{D}_{M+1}^{l}$, and that $l<0$ since if $l \geq 0$ then $\mathcal{D}_{M}^{l} \subset \mathcal{D}_{M}^{-l}$. Thus $\widetilde{\varphi} \in \mathcal{D}_{M}^{-k}\left(\mathbb{Q}_{p}^{n}\right)$ with $M$, $k \geq 1$, and $\mathcal{D}_{M}^{-k}\left(\mathbb{Q}_{p}^{n}\right) \subset \mathcal{D}_{\max \{k, M\}}^{-\max \{k, M\}}\left(\mathbb{Q}_{p}^{n}\right) \subset \mathcal{D}_{N}^{-N}\left(\mathbb{Q}_{p}^{n}\right)$ for $N \geq \max \{k, M\}$, i.e. $\widetilde{\varphi} \in \mathcal{D}_{N}^{-N}\left(\mathbb{Q}_{p}^{n}\right)$ for $N \geq \max \{k, M\}$, and $\widetilde{\varphi}(x)=\sum_{\boldsymbol{i} \in G_{N}^{n}} \widetilde{\varphi}(\boldsymbol{i}) \Omega\left(p^{M}\|x-\boldsymbol{i}\|_{p}\right)=$ $P_{N} \widetilde{\varphi}(x)$. Now

$$
\left\|\varphi-P_{N} \varphi\right\|_{\infty} \leq\|\varphi-\widetilde{\varphi}\|_{\infty}+\left\|\widetilde{\varphi}-P_{N} \varphi\right\|_{\infty} \leq \epsilon+\left\|P_{N} \widetilde{\varphi}-P_{N} \varphi\right\|_{\infty} \leq 2 \epsilon
$$

for $N \geq \max \{k, M\}$, since $\left\|P_{N} \widetilde{\varphi}-P_{N} \varphi\right\|_{\infty}=\sup _{\boldsymbol{i}}|\widetilde{\varphi}(\boldsymbol{i})-\varphi(\boldsymbol{i})| \leq\|\widetilde{\varphi}-\varphi\|_{\infty}<\epsilon$ for $N \geq \max \{k, M\}$.

We denote by $E_{N}, N \geq 1$, the embedding $X_{N} \rightarrow X_{\infty}$. The following result is a consequence of the above observations. If $Z, Y$ are real Banach spaces, we denote by $\mathfrak{B}(Z, Y)$, the space of all linear bounded operators from $Z$ into $Y$.

Lemma 2 (Condition A). With the above notation, the following assertions hold: (i) $X_{\infty}, X_{N}$ for $N \geq 1$, are real Banach spaces, all with the norm $\|\cdot\|_{\infty}$;

(ii) $P_{N} \in \mathfrak{B}\left(X_{\infty}, X_{N}\right)$ and $\left\|P_{N} \varphi\right\|_{\infty} \leq\|\varphi\|_{\infty}$ for any $N \geq 1, \varphi \in X_{\infty}$;

(iii) $E_{N} \in \mathfrak{B}\left(X_{N}, X_{\infty}\right)$ and $\left\|E_{N} \varphi\right\|_{\infty}=\|\varphi\|_{\infty}$ for any $N \geq 1, \varphi \in X_{N}$;

(iv) $P_{N} E_{N} \varphi=\varphi$ for $N \geq 1, \varphi \in X_{N}$.

3.1. The operators $A_{N}, A$. Set $\mathbb{R}_{+}:=\{x \in \mathbb{R} ; x \geq 0\}$. We fix a continuous function $J: \mathbb{R}_{+} \rightarrow \mathbb{R}_{+}$, and take $J(x)=J\left(\|x\|_{p}\right)$ for $x \in \mathbb{Q}_{p}^{n}$, then $J(x)$ is a radial function on $\mathbb{Q}_{p}^{n}$. In addition, we assume that $\int_{\mathbb{Q}_{p}^{n}} J\left(\|x\|_{p}\right) d^{n} x=1$.

Lemma 3. The following assertions hold:

(i) set $J_{N}\left(\|x\|_{p}\right):=J\left(\|x\|_{p}\right) \Omega\left(p^{-N}\|x\|_{p}\right)$ for $N \geq 1$. Then

$$
J_{N}\left(\|x\|_{p}\right) * P_{N} \varphi(x)=\Omega\left(p^{-N}\|x\|_{p}\right)\left\{J\left(\|x\|_{p}\right) * P_{N} \varphi(x)\right\}
$$

for $\varphi(x) \in X_{\infty}$

(ii) Define for $N \geq 1$,

$$
\begin{aligned}
A_{N}: & X_{N} \\
\phi(x) & \rightarrow-X_{N} \\
B_{N}^{n} & J_{N}\left(\|x-y\|_{p}\right)\{\phi(y)-\phi(x)\} d^{n} y .
\end{aligned}
$$

Then $A_{N}$ is a well-defined linear bounded operator.

Proof. (i) We recall that $B_{N}^{n}(0)=B_{N}^{n}$. Notice that

$$
\begin{aligned}
I(x) & :=J_{N}\left(\|x\|_{p}\right) * P_{N} \varphi(x)=\int_{\mathbb{Q}_{p}^{n}} J\left(\|x-y\|_{p}\right) \Omega\left(p^{-N}\|x-y\|_{p}\right) P_{N} \varphi(y) d^{n} y \\
& =\int_{B_{N}^{n}(0) \cap B_{N}^{n}(x)} J\left(\|x-y\|_{p}\right) P_{N} \varphi(y) d^{n} y .
\end{aligned}
$$

The calculation of the above integral involves two cases: (1) $B_{N}^{n}(0) \cap B_{N}^{n}(x) \neq \varnothing$; (2) $B_{N}^{n}(0) \cap B_{N}^{n}(x)=\varnothing$. In the first case, since the radii of the balls are the same, 
$B_{N}^{n}(0)=B_{N}^{n}(x)$ and thus $x \in B_{N}^{n}(0)$ which implies that $\|x\|_{p} \leq p^{N}$, in addition, $\operatorname{supp} I(x) \subset B_{N}^{n}(0)$, and thus

$$
\begin{aligned}
I(x) & =\Omega\left(p^{-N}\|x\|_{p}\right) \int_{B_{N}^{n}(0)} J\left(\|x-y\|_{p}\right) P_{N} \varphi(y) d^{n} y \\
& =\Omega\left(p^{-N}\|x\|_{p}\right) \int_{\mathbb{Q}_{p}^{n}} J\left(\|x-y\|_{p}\right) P_{N} \varphi(y) d^{n} y .
\end{aligned}
$$

In the second case, $x \notin B_{N}^{n}(0)$ this implies that $\|x\|_{p}>p^{-N}$, and $I(x)=0$.

(ii) Notice that

$$
A_{N} \phi(x)=-\left\{J_{N}\left(\|x\|_{p}\right) * \phi(x)-j_{N} \phi(x)\right\}, \text { with } j_{N}:=\int_{B_{N}^{n}} J\left(\|y\|_{p}\right) d^{n} y,
$$

$0 \leq j_{N} \leq 1$ and that

$$
\lim _{N \rightarrow \infty} j_{N}=1 .
$$

From (3.2) and part (i), it follows that the operator is well-defined. Indeed, for $\phi \in$ $X_{N}, \operatorname{supp}\left(J_{N}\left(\|x\|_{p}\right) * \phi(x)\right) \subset B_{N}^{n}$ and the index of local constancy of $J_{N}\left(\|x\|_{p}\right) *$ $\phi(x)$ equals the index of local constancy of $\phi$. The continuity follows from the Young inequality:

$$
\left|A_{N} \phi(x)\right| \leq\left\|J_{N}\left(\|x\| \|_{p}\right)\right\|_{L^{1}}\|\phi(x)\|_{\infty}+j_{N}\|\phi(x)\|_{\infty} \leq 2\|\phi(x)\|_{\infty} .
$$

Now, we define

$$
\begin{aligned}
A: X_{\infty} & \rightarrow X_{\infty} \\
\varphi(x) & \rightarrow A \varphi(x)=-\left\{J\left(\|x\|_{p}\right) * \varphi(x)-\varphi(x)\right\} .
\end{aligned}
$$

Remark 1. Notice that $A \varphi(x)=-\int_{\mathbb{Q}_{p}^{n}} J\left(\|x-y\|_{p}\right)\{\varphi(y)-\varphi(x)\} d^{n} y$ since $\int_{\mathbb{Q}_{p}^{n}} J\left(\|x-y\|_{p}\right) d^{n} y=1$.

Lemma 4. The operator $A: X_{\infty} \rightarrow X_{\infty}$ is a linear and bounded. In addition, the spectrum of $A, \sigma(A)$, is contained in the interval $[0,2]$.

Proof. By the Young inequality, $A \in \mathfrak{B}\left(X_{\infty}, L^{\infty}\right)$. Now, by construction $\mathcal{D}\left(\mathbb{Q}_{p}^{n}\right)$ is dense in $X_{\infty}\left(\mathbb{Q}_{p}^{n}\right)$ with respect to $\|\cdot\|_{\infty}$, then in order to show that $A$ is densely defined and continuous, from $X_{\infty}$ into itself, it is sufficient to show that $A \varphi \in X_{\infty}$ for $\varphi \in \mathcal{D}_{N}^{-N} \subset \mathcal{D}\left(\mathbb{Q}_{p}^{n}\right)$. Thus, we have to show that $A \varphi \in X_{\infty}$ for $\varphi \in X_{N}$. By Lemma 3, $\Omega\left(p^{-N}\|x\|_{p}\right) A \varphi=A_{N} \varphi \in X_{N}$, now we show that $A_{N} \varphi\|\cdot\|_{\infty} A \varphi$ for $\varphi \in X_{N}$. In order to achieve this, by Lemma 1, it is sufficient to show that $J_{N}\left(\|x\|_{p}\right) * \varphi(x) \stackrel{\|\cdot\|_{\infty}}{\longrightarrow} J\left(\|x\|_{p}\right) * \varphi(x)$ for $\varphi \in X_{N}$. Indeed,

$$
\left\|\left\{J\left(\|x\|_{p}\right)-J_{N}\left(\|x\|_{p}\right)\right\} * \varphi(x)\right\|_{\infty} \leq\|\varphi\|_{\infty} \int_{\mathbb{Q}_{p}^{n}}\left|J\left(\|y\|_{p}\right)-J_{N}\left(\|y\|_{p}\right)\right| d^{n} y .
$$

This last integral tends to zero as $N$ tends to infinity by the Dominated Convergence Theorem, recall that $\int_{\mathbb{Q}_{p}^{n}} J_{N}\left(\|y\|_{p}\right) d^{n} y \leq \int_{\mathbb{Q}_{p}^{n}} J\left(\|y\|_{p}\right) d^{n} y=1$. 
The comment about the spectrum of $A$ follows from the following observation: the equation $A \varphi=\lambda \varphi$ is equivalent to $J * \varphi=(1-\lambda) \varphi$ and since $\|J * \cdot\| \leq 1$, we have $0 \leq \lambda \leq 2$.

\section{The Matrix Representation of operators $A_{N}$ and Markov Chains}

By using the basis $\left\{\Omega\left(p^{N}\|x-i\|_{p}\right)\right\}_{i \in G_{N}^{n}}$, we identify $X_{N}$ with $\left(\mathbb{R}^{\# G_{N}^{n}},\|\cdot\|_{\mathbb{R}}\right)$, thus operator $A_{N}$ is given by a matrix. This matrix is computed by means of the following two lemmas.

Lemma 5. Set $\mathfrak{a}(x, \boldsymbol{i}):=J_{N}\left(\|x\|_{p}\right) * \Omega\left(p^{N}\|x-\boldsymbol{i}\|_{p}\right)$ for $x \in B_{N}^{n}, \boldsymbol{i} \in G_{N}^{n}$. Let $\widetilde{x}$ denote the image of $x$ under the canonical map $B_{N}^{n} \rightarrow G_{N}^{n}$. Then

$$
\mathfrak{a}(x, \boldsymbol{i})=\mathfrak{a}(\widetilde{x}, \boldsymbol{i})=\left\{\begin{array}{ccc}
p^{-N n} J\left(p^{-\operatorname{ord}(\widetilde{x}-\boldsymbol{i})}\right) & \text { if } & \operatorname{ord}(\widetilde{x}-\boldsymbol{i}) \neq+\infty \\
\int_{\left(p^{N} \mathbb{Z}_{p}\right)^{n}} J\left(\|y\|_{p}\right) d^{n} y & \text { if } & \operatorname{ord}(\widetilde{x}-\boldsymbol{i})=+\infty .
\end{array}\right.
$$

Proof. We first notice that since $\widetilde{x}-\boldsymbol{i}+\left(p^{N} \mathbb{Z}_{p}\right)^{n} \subset B_{N}^{n}$, it verifies that

$$
\begin{aligned}
\mathfrak{a}(x, \boldsymbol{i}) & =\int_{x-\boldsymbol{i}+\left(p^{N} \mathbb{Z}_{p}\right)^{n}} J_{N}\left(\|y\|_{p}\right) d^{n} y=\int_{\widetilde{x}-\boldsymbol{i}+\left(p^{N} \mathbb{Z}_{p}\right)^{n}} J_{N}\left(\|y\|_{p}\right) d^{n} y \\
& =\int_{\widetilde{x}-\boldsymbol{i}+\left(p^{N} \mathbb{Z}_{p}\right)^{n}} J\left(\|y\|_{p}\right) d^{n} y=J\left(\|\widetilde{x}\|_{p}\right) * \Omega\left(p^{N}\|\widetilde{x}-\boldsymbol{i}\|_{p}\right) \\
& =\int_{\left(p^{N} \mathbb{Z}_{p}\right)^{n}} J\left(\|\widetilde{x}-\boldsymbol{i}-y\|_{p}\right) d^{n} y .
\end{aligned}
$$

On the other hand, since $\operatorname{ord}\left(G_{N}^{n}\right)=\{-N,-N+1, \ldots, 0,1, \ldots, N-1,+\infty\}$ and $\operatorname{ord}(y) \geq N$ for $y \in\left(p^{N} \mathbb{Z}_{p}\right)^{n}$, then $\|\widetilde{x}-\boldsymbol{i}-y\|_{p}=\|\widetilde{x}-\boldsymbol{i}\|_{p}$ if and only if $\operatorname{ord}(\widetilde{x}-\boldsymbol{i}) \neq$ $+\infty$. The announced formula now follows from (4.1).

Remark 2. Notice that $\mathfrak{a}(\widetilde{x}, \boldsymbol{i})=\mathfrak{a}(\boldsymbol{i}, \widetilde{x})=\mathfrak{a}\left(\|\widetilde{x}-\boldsymbol{i}\|_{p}\right)$, where $\mathfrak{a}\left(\|\widetilde{x}-\boldsymbol{i}\|_{p}\right)$ means that there exists a function $g: \mathbb{R}_{+} \rightarrow \mathbb{R}$ such that $\mathfrak{a}(\boldsymbol{i}, \widetilde{x})=g\left(\|\widetilde{x}-\boldsymbol{i}\|_{p}\right)$, i.e. $\mathfrak{a}(\boldsymbol{i}, \widetilde{x})$ is a radial function of $\widetilde{x}-\boldsymbol{i}$.

Lemma 6. The matrix for operator $A_{N}$ acting on $X_{N}$ is $A^{(N)}=\left[A_{\boldsymbol{k i}}^{(N)}\right]_{\boldsymbol{k}, \boldsymbol{i} \in G_{N}^{n}}=$ $\left[j_{N} \delta_{\boldsymbol{k} i}-\mathfrak{a}_{\boldsymbol{k i}}\right]_{\boldsymbol{k}, \boldsymbol{i} \in G_{N}^{n}}$, where $\mathfrak{a}_{\boldsymbol{k} \boldsymbol{i}}:=\mathfrak{a}(\boldsymbol{k}, \boldsymbol{i})$ and $\delta_{\boldsymbol{k} \boldsymbol{i}}$ denotes the Kronecker delta.

Proof. Notice that $\left\{\Omega\left(p^{N}\|x-i\|_{p}\right)\right\}_{\boldsymbol{i} \in G_{N}^{n}}$ is an orthogonal basis of $X_{N}$ under the usual inner product of real-valued functions, which is denoted as $\langle\cdot, \cdot\rangle$, since

$$
\Omega\left(p^{N}\|x-\boldsymbol{i}\|_{p}\right) \Omega\left(p^{N}\|x-\boldsymbol{j}\|_{p}\right) \equiv 0 \text { if } \boldsymbol{i} \neq \boldsymbol{j} .
$$

Take $\varphi(x)=\sum_{\boldsymbol{i} \in G_{N}^{n}} \varphi(\boldsymbol{i}) \Omega\left(p^{N}\|x-\boldsymbol{i}\|_{p}\right)$, then

$$
J_{N}\left(\|x\|_{p}\right) * \Omega\left(p^{N}\|x-i\|_{p}\right)=\sum_{\boldsymbol{k} \in G_{N}^{n}} d_{\boldsymbol{k} i} \Omega\left(p^{N}\|x-\boldsymbol{k}\|_{p}\right),
$$


with $d_{\boldsymbol{k} i} \in \mathbb{R}$, cf. Lemma 3 , and

$$
A_{N} \varphi(x)=\sum_{\boldsymbol{k} \in G_{N}^{n}}\left\{\sum_{\boldsymbol{i} \in G_{N}^{n}}\left(j_{N} \delta_{\boldsymbol{k} \boldsymbol{i}}-d_{\boldsymbol{k} \boldsymbol{i}}\right) \varphi(\boldsymbol{i})\right\} \Omega\left(p^{N}\|x-\boldsymbol{k}\|_{p}\right) .
$$

Hence $\left[j_{N} \delta_{\boldsymbol{k} i}-d_{\boldsymbol{k} \boldsymbol{i}}\right]_{\boldsymbol{k}, \boldsymbol{i} \in G_{N}^{n}}$ is the matrix representation for operator $A_{N}$ acting on $X_{N}$. To compute the coefficients $d_{\boldsymbol{k} i}$ we proceed as follows. By using that

$$
\Omega\left(p^{N}\|y-\boldsymbol{j}\|_{p}\right) * \Omega\left(p^{N}\|y-\boldsymbol{l}\|_{p}\right)=p^{-N n} \Omega\left(p^{N}\|y-(\boldsymbol{j}+\boldsymbol{l})\|_{p}\right),
$$

we have

$$
\begin{gathered}
d_{\boldsymbol{k i}}=P^{N n}\left\langle J_{N}\left(\|x\|_{p}\right) * \Omega\left(p^{N}\|x-\boldsymbol{i}\|_{p}\right), \Omega\left(p^{N}\|x-\boldsymbol{k}\|_{p}\right)\right\rangle \\
=p^{N n} \int_{\mathbb{Q}_{p}^{n}} \int_{\mathbb{Q}_{p}^{n}} J_{N}\left(\|y\|_{p}\right) \Omega\left(p^{N}\|x-\boldsymbol{i}-y\|_{p}\right) \Omega\left(p^{N}\|x-\boldsymbol{k}\|_{p}\right) d^{n} y d^{n} x \\
=p^{N n} \int_{\mathbb{Q}_{p}^{n}} J_{N}\left(\|y\|_{p}\right) \Omega\left(p^{N}\|y+\boldsymbol{i}\|_{p}\right) * \Omega\left(p^{N}\|y-\boldsymbol{k}\|_{p}\right) d^{n} y \\
=\int_{\mathbb{Q}_{p}^{n}} J_{N}\left(\|y\|_{p}\right) \Omega\left(p^{N}\|y-(\boldsymbol{k}-\boldsymbol{i})\|_{p}\right) d^{n} y=\int_{\boldsymbol{k}-\boldsymbol{i}+\left(p^{N} \mathbb{Z}_{p}\right)^{n}} J\left(\|y\|_{p}\right) d^{n} y \\
=\mathfrak{a}(\boldsymbol{k}, \boldsymbol{i}),
\end{gathered}
$$

cf. Lemma 5 .

Lemma 7. $-A^{(N)}$ is a $Q$-matrix, i.e. $-A_{\boldsymbol{i j}}^{(N)} \geq 0$ for $\boldsymbol{i} \neq \boldsymbol{j}$ with $\boldsymbol{i}, \boldsymbol{j} \in G_{N}^{n}$, and $A_{\boldsymbol{i} \boldsymbol{i}}^{(N)}=-\sum_{\boldsymbol{j} \neq \boldsymbol{i}} A_{\boldsymbol{i j}}^{(N)}$.

Proof. We first notice that

$$
j_{N}-\mathfrak{a}_{i \boldsymbol{i}}=\int_{B_{N}^{n}} J\left(\|y\|_{p}\right) d^{n} y-\int_{B_{-N}^{n}} J\left(\|y\|_{p}\right) d^{n} y \geq 0,
$$

cf. Lemmas 6. 5. Now, $A_{\boldsymbol{i} \boldsymbol{j}}^{(N)}=-\mathfrak{a}_{\boldsymbol{i j}}$ for $\boldsymbol{j} \neq \boldsymbol{i}$ with $\mathfrak{a}_{\boldsymbol{i} \boldsymbol{j}} \geq 0$, and by using that $G_{N}^{n}$ is an additive group, and that $B_{N}^{n}=\coprod_{\boldsymbol{k} \in G_{N}^{n}} B_{-N}^{n}(\boldsymbol{k})$, we have

$$
\begin{aligned}
\sum_{\boldsymbol{j} \neq \boldsymbol{i}} \mathfrak{a}_{i \boldsymbol{j}} & =\sum_{\boldsymbol{j} \neq \boldsymbol{i}} \int_{B_{-N}^{n}(\boldsymbol{j}-\boldsymbol{i})} J\left(\|y\|_{p}\right) d^{n} y=\sum_{\boldsymbol{k} \neq \mathbf{0}_{B_{-N}^{n}}(\boldsymbol{k})} \int_{B_{N}^{n}} J\left(\|y\|_{p}\right) d^{n} y \\
& =\int_{B_{-N}^{n}} J\left(\|y\|_{p}\right) d^{n} y-\int_{\|} J\left(\|y\|_{p}\right) d^{n} y=j_{N}-\mathfrak{a}_{\boldsymbol{i} i}
\end{aligned}
$$

i.e. $A_{\boldsymbol{i} \boldsymbol{i}}^{(N)}=-\sum_{\boldsymbol{j} \neq \boldsymbol{i}} A_{\boldsymbol{i} \boldsymbol{j}}^{(N)}$.

A real matrix $A$ is called nonnegative if each of its entries is greater than or equal to zero, in this case, we use the notation $A \geq \mathbf{0}$. Similarly, we say that a real matrix is nonpositive if each of its entries is less than or equal to zero, in this case, we use the notation $A \leq \mathbf{0}$. We denote by $\mathbb{E}$ the identity matrix and by $\mathbf{1}$ the unit vector, which is the vector having all its entries equal to one. 
Theorem 1. (i) Set $P^{(N)}(t):=e^{-t A^{(N)}}, t \geq 0$. Then $P^{(N)}(t)$ is a semigroup of nonnegative matrices, with $P^{(N)}(0)=\mathbb{E}$, satisfying

$$
\frac{\partial P^{(N)}(t)}{\partial t}+A^{(N)} P^{(N)}(t)=0
$$

and $P^{(N)}(t) \mathbf{1}=\mathbf{1}$ for $t \geq 0$.

(ii) The function $P^{(N)}(t-s), t \geq s \geq 0$, is the transition function of a homogeneous Markov chain with state space $G_{N}^{n}$. Furthermore, this stochastic process has right-continuous piece-wise-constant paths.

Proof. The result follows from Lemma 7 by using well-known results about Markov chains, see e.g. [37, Theorem 2.5].

\section{Non-Archimedean Helmholtz Free-Energy Functionals}

$$
E_{N}(\varphi)=\frac{1}{4} \int_{B_{N}^{n}} \int_{B_{N}^{n}} J_{N}\left(\|x-y\|_{p}\right)\{\varphi(x)-\varphi(y)\}^{2} d^{n} x d^{n} y+\lambda \int_{B_{N}^{n}} W(\varphi(x)) d^{n} x,
$$

where $J_{N}\left(\|x\|_{p}\right)$ is as before, $\varphi$ is a scalar density function defined on $B_{N}^{n}$ that takes values in $[-1,1], W: \mathbb{R} \rightarrow \mathbb{R}$, with derivative $f \in C^{2}(\mathbb{R})$, is a double-well potential having (not necessarily equal) minima at \pm 1 . The functional $E_{N}(\varphi)$ is a non-Archimedean version of a non-local Helmholtz free-energy functional. The function $\varphi$, the order parameter, represents the macroscopic density profile of a system which has two equilibrium pure phases described by the profiles $\varphi \equiv 1$ and $\varphi \equiv-1$, and $-1<\varphi<1$ represents the 'interface'. The function $J_{N}$ is a positive, possibly anisotropic, interaction potential which vanishes at infinity. If $\varphi$ is an energy minimizing configuration, the second term in $E_{N}$ forces the minimizer $\varphi$ to take values close the pure states, while the first term in $E_{N}$ represents an interaction energy which penalizes the spatial inhomogenety of $\varphi$.

In the classical Archimedean setting (i.e. $\mathbb{R}^{n}$ ), the $L^{2}$-gradient of functionals of type (5.1) lead to the non-local versions of Allen-Cahn equations, see [2]-[3], 12] The next result shows that a similar situation happens in the non-Archimedean setting.

Lemma 8. (i) By identifying $\varphi(x)$ with the vector $[\varphi(\boldsymbol{i})]_{\boldsymbol{i} \in G_{N}^{n}}$, i.e. by identifying $X_{N}$ with $\mathbb{R}^{\# G_{N}^{n}}$, we have

$$
\begin{aligned}
E_{N}\left([\varphi(\boldsymbol{i})]_{\boldsymbol{i} \in G_{N}^{n}}\right) & =\frac{j_{N} p^{-N n}}{2} \sum_{\boldsymbol{i} \in G_{N}^{n}} \varphi^{2}(\boldsymbol{i})-\frac{p^{-N n}}{2} \sum_{\boldsymbol{i}, \boldsymbol{j} \in G_{N}^{n}} \mathfrak{a}_{i \boldsymbol{j}} \varphi(\boldsymbol{i}) \varphi(\boldsymbol{j}) \\
& +\lambda p^{-N n} \sum_{\boldsymbol{i} \in G_{N}^{n}} W(\varphi(\boldsymbol{i})),
\end{aligned}
$$

where $\left[\mathfrak{a}_{i j}\right]_{\boldsymbol{i}, \boldsymbol{j} \in G_{N}^{n}}$ is the matrix defined in Lemma 占.

(ii) We assume that $\varphi$ depends on $\boldsymbol{i} \in G_{N}^{n}$ and $t \geq 0$. The gradient flow in the Euclidean space $\mathbb{R}^{\# G_{N}^{n}}$ of the functional $E_{N}: \mathbb{R}^{\# G_{N}^{n}} \rightarrow \mathbb{R}$ is the evolution in $\mathbb{R}^{\# G_{N}^{n}}$ 
given by

$$
\begin{aligned}
\frac{\partial}{\partial t}[\varphi(\boldsymbol{i}, t)]_{i \in G_{N}^{n}} & =-\nabla E_{N}\left([\varphi(\boldsymbol{i}, t)]_{i \in G_{N}^{n}}\right) \\
& =-p^{-N n} A^{(N)}[\varphi(\boldsymbol{i}, t)]_{i \in G_{N}^{n}}-\lambda p^{-N n}[f(\varphi(\boldsymbol{i}, t))]_{i \in G_{N}^{n}},
\end{aligned}
$$

where $A^{(N)}$ is the matrix defined in Lemma 6 .

Remark 3. Notice that in $X_{N}$, (5.2) can be written as

$$
\frac{\partial}{\partial t} \varphi(x, t)=-A_{N} \varphi(x, t)-\lambda f(\varphi(x, t)) .
$$

Proof. (i) By using that $\varphi^{2}(x)=\sum_{\boldsymbol{i} \in G_{N}^{n}} \varphi^{2}(i) \Omega\left(p^{N}\|x-i\|_{p}\right), \varphi(x) \varphi(y)=$ $\sum_{\boldsymbol{i}, \boldsymbol{j} \in G_{N}^{n}} \varphi(\boldsymbol{i}) \varphi(\boldsymbol{j}) \Omega\left(p^{N}\|x-\boldsymbol{i}\|_{p}\right) \Omega\left(p^{N}\|y-\boldsymbol{j}\|_{p}\right)$, we have

$$
\begin{gathered}
E_{N}\left(\left[\varphi_{\boldsymbol{i}}\right]_{\boldsymbol{i} \in G_{N}^{n}}\right)=\frac{1}{2} \sum_{\boldsymbol{i} \in G_{N}^{n}} \varphi^{2}(\boldsymbol{i}) \int_{B_{N}^{n}} \int_{B_{N}^{n}} J\left(\|x-y\|_{p}\right) \Omega\left(p^{N}\|x-\boldsymbol{i}\|_{p}\right) d^{n} x d^{n} y \\
-\frac{1}{2} \sum_{\boldsymbol{i}, \boldsymbol{j} \in G_{N}^{n}} \varphi(\boldsymbol{i}) \varphi(\boldsymbol{j}) \int_{B_{N}^{n}} \int_{B_{N}^{n}} J\left(\|x-y\|_{p}\right) \Omega\left(p^{N}\|x-\boldsymbol{i}\|_{p}\right) \Omega\left(p^{N}\|y-\boldsymbol{j}\|_{p}\right) d^{n} x d^{n} y \\
+\lambda \sum_{\boldsymbol{i} \in G_{N}^{n}} W(\varphi(\boldsymbol{i})) \int_{B_{N}^{n}} \Omega\left(p^{N}\|x-\boldsymbol{i}\|_{p}\right) d^{n} x .
\end{gathered}
$$

The announced formula follows from the following observations:

$$
\begin{aligned}
& \int_{B_{N}^{n}} \Omega\left(p^{N}\|x-i\|_{p}\right)\left\{\int_{B_{N}^{n}} J\left(\|x-y\|_{p}\right) d^{n} y\right\} d^{n} x \\
& =\int_{B_{N}^{n}} \Omega\left(p^{N}\|x-i\|_{p}\right)\left\{\int_{B_{N}^{n}} J\left(\|z\|_{p}\right) d^{n} z\right\} d^{n} x=p^{-N n} j_{N},
\end{aligned}
$$

and

$$
\begin{aligned}
& \int_{B_{N}^{n}} \int_{B_{N}^{n}} J\left(\|x-y\|_{p}\right) \Omega\left(p^{N}\|x-\boldsymbol{i}\|_{p}\right) \Omega\left(p^{N}\|y-\boldsymbol{j}\|_{p}\right) d^{n} x d^{n} y \\
& =\left\langle J\left(\|x\|_{p}\right) * \Omega\left(p^{N}\|x-\boldsymbol{i}\|_{p}\right), \Omega\left(p^{N}\|x-\boldsymbol{j}\|_{p}\right)\right\rangle=p^{-N n} \mathfrak{a}_{\boldsymbol{i j}},
\end{aligned}
$$

see (4.2).

(ii) By using the first part, with $\langle\cdot, \cdot\rangle_{\mathbb{R}}$ denoting the inner product in $\mathbb{R}^{\# G_{N}^{n}}$, the directional derivative (i.e. functional derivative) of $E_{N}$ is given by

$$
\begin{aligned}
& \lim _{\epsilon \rightarrow 0} \frac{E_{N}\left([\varphi(\boldsymbol{i})+\epsilon \theta(\boldsymbol{i})]_{\boldsymbol{i} \in G_{N}^{n}}\right)-E_{N}\left([\varphi(\boldsymbol{i})]_{\boldsymbol{i} \in G_{N}^{n}}\right)}{\epsilon} \\
&=j_{N} p^{-N n} \sum_{\boldsymbol{i}} \varphi(\boldsymbol{i}) \theta(\boldsymbol{i})-p^{-N n} \sum_{\boldsymbol{i}, \boldsymbol{j}} \mathfrak{a}_{\boldsymbol{i j}} \varphi(\boldsymbol{j}) \theta(\boldsymbol{i})+\lambda p^{-N n} \sum_{\boldsymbol{i}} f(\varphi(\boldsymbol{i})) \theta(\boldsymbol{i}) \\
&=p^{-N n}\left\langle A^{(N)}[\varphi(\boldsymbol{i})]_{\boldsymbol{i} \in G_{N}^{n}}+\lambda[f(\varphi(\boldsymbol{i}))]_{\boldsymbol{i} \in G_{N}^{n}},[\theta(\boldsymbol{i})]_{\boldsymbol{i} \in G_{N}^{n}}\right\rangle_{\mathbb{R}},
\end{aligned}
$$


i.e. $\nabla E_{N}\left([\varphi(i)]_{i \in G_{N}^{n}}\right)=p^{-N n}\left(A^{(N)}[\varphi(\boldsymbol{i})]_{i \in G_{N}^{n}}+\lambda[f(\varphi(\boldsymbol{i}))]_{\boldsymbol{i} \in G_{N}^{n}}\right)$ where $\nabla g$ denotes the standard gradient vector in $\mathbb{R}^{\# G_{N}^{n}}$. On the other hand, in the space $X_{N}$, we have $\nabla E_{N}(\varphi)=A_{N} \varphi+\lambda f(\varphi)$ since

$$
\begin{aligned}
& p^{-N n}\left\langle A^{(N)}[\varphi(\boldsymbol{i})]_{\boldsymbol{i} \in G_{N}^{n}}+\lambda[f(\varphi(\boldsymbol{i}))]_{\boldsymbol{i} \in G_{N}^{n}},[\theta(\boldsymbol{i})]_{\boldsymbol{i} \in G_{N}^{n}}\right\rangle_{\mathbb{R}} \\
& =\int_{\mathbb{Q}_{p}^{n}}\left\{A_{N} \varphi(x)+\lambda f(\varphi(x))\right\} \theta(x) d^{n} x
\end{aligned}
$$

in $X_{N}$.

Consider $\left(G_{N}^{n},\|\cdot\|_{p}\right)$ as a finite ultrametric space. Then (5.2) is reaction-ultradiffusion equation in $\left(G_{N}^{n},\|\cdot\|_{p}\right)$, which is the $L^{2}$-gradient of an energy functional defined on $\left(G_{N}^{n},\|\cdot\|_{p}\right)$. These equations are generalizations of the ultradiffusion equations studied in [30] and [10]. In this article we initiate the study of these equations and their 'limits' as $N$ tends to infinity. The limit of some ultradiffusion equations of type (5.2) with $f \equiv 0$ was considered by Avetisov et al. in [9, when the matrix $A^{(N)}$ comes from a Parisi matrix. More precisely, in [9] was established, by using a physical argument, that the 'limit' of an equation of type (5.2) as $N$ tends to infinity is

$$
\frac{\partial}{\partial t} \varphi(x, t)=-A \varphi(x, t)-\lambda f(\varphi(x, t)), x \in \mathbb{Q}_{p}^{n}, t \geq 0 .
$$

In this article we show, from a mathematical perspective, that the solutions of the Cauchy problem attached to the equation (5.3) converge to the solutions of the Cauchy problem attached to the equation (5.4), see Theorem 7 , in the case that $f$ $\in C^{2}$ with three zeros at $-1,0,1$. The equation (5.4) is formally the $L^{2}$-gradient of the following energy functional:

$$
E(\varphi)=\frac{1}{4} \int_{\mathbb{Q}_{p}^{n}} \int_{\mathbb{Q}_{p}^{n}} J\left(\|x-y\|_{p}\right)\{\varphi(x)-\varphi(y)\}^{2} d^{n} x d^{n} y+\lambda \int_{\mathbb{Q}_{p}^{n}} W(\varphi(x)) d^{n} x,
$$

where $\varphi$ is a scalar density function defined on $\mathbb{Q}_{p}^{n}$ that takes values in $[-1,1], W$ is a double-well potential having minima at \pm 1 as before. At the moment, we do not know if $E(\varphi)$ can be well approximated by $E_{N}\left(P_{N} \varphi\right)$ for $\varphi \in X_{\infty}$. On the other hand, if supp $J \subset K$, open and compact, with $K \subset B_{N_{0}}^{n}$, and $W^{\prime}=f$ is continuous, then for any $\varphi \in X_{\infty}(K)$, and $N \geq N_{0}$, the functional

$$
\begin{aligned}
E_{N}\left(P_{N} \varphi\right) & =\frac{1}{4} \int_{K} \int_{K} J\left(\|x-y\|_{p}\right)\left\{P_{N} \varphi(x)-P_{N} \varphi(y)\right\}^{2} d^{n} x d^{n} y \\
& +\lambda \int_{K} W\left(P_{N} \varphi(x)\right) d^{n} x
\end{aligned}
$$

tends to

$$
\frac{1}{4} \int_{K} \int_{K} J\left(\|x-y\|_{p}\right)\{\varphi(x)-\varphi(y)\}^{2} d^{n} x d^{n} y+\lambda \int_{K} W(\varphi(x)) d^{n} x
$$

as $N$ tends to infinity. The verification of this assertion follows directly from the Dominated Convergence Theorem and the fact that $P_{N} \varphi \stackrel{\|\cdot\|_{\infty}}{\longrightarrow}$. 


\section{Stationary Solutions}

We take $J(x)=J\left(\|x\|_{p}\right)$ for $x \in \mathbb{Q}_{p}^{n}$ as in Section 3.1. We fix a function $f: \mathbb{R} \rightarrow \mathbb{R}$ having the following properties:

$$
f \in C^{2}(\mathbb{R})
$$

$$
f \text { has exactly three zeros at }-1,0,1 \text {; }
$$

$$
f^{\prime}(-1)>0, f^{\prime}(0)<0, f^{\prime}(1)>0
$$

(H4) the function $g(u):=u+\lambda f(u)$ has three zeros and exactly three intervals of monotonicity for any sufficiently large $\lambda>0$. We denote by $u_{\lambda}^{-}, u_{\lambda}^{+}$, the extreme roots of $g(u)=0$, then $u_{\lambda}^{-}<u_{\lambda}^{+}, u_{\lambda}^{-}>-1, u_{\lambda}^{+}<1$, and $u_{\lambda}^{ \pm} \rightarrow \pm 1$ as $\lambda \rightarrow \infty$.

The following technical conditions always hold under Hypotheses (H1)-(H4) by taking $\lambda$ sufficiently large:

(C5) We take $\alpha^{-} \in(-1,0), \alpha^{+} \in(0,1)$ such that $f^{\prime}(u) \geq \delta>0$ for $u$ in $\left[-1, \alpha^{-}\right] \cup$ $\left[\alpha^{+}, 1\right]$. In addition, we assume that $\alpha^{+}, \alpha^{-}$satisfy $u_{\lambda}^{-}<\alpha^{-}<0<\alpha^{+}<u_{\lambda}^{+}$;

$$
\begin{gathered}
\left(1+\alpha^{+}\right)+\lambda f\left(\alpha^{+}\right) \leq 0 ; \\
\alpha^{-}+\lambda f\left(\alpha^{-}\right) \geq 0 .
\end{gathered}
$$

Notice that conditions C6-C7 hold if $\lambda \geq \max \left\{\frac{-\alpha^{-}}{f\left(\alpha^{-}\right)}, \frac{1+\alpha^{+}}{-f\left(\alpha^{+}\right)}\right\}$.

Remark 4. Notice that polynomial $u^{3}-u$ satisfies hypotheses H1-H4. If $f(u) \in$ $C^{2}(\mathbb{R})$ and $f^{\prime \prime}(u)$ has exactly a zero $\beta \in(-1,1)$ and $f^{\prime \prime}(u)$ is positive to the right of $\beta$ and negative to the left of $\beta$, then $g(u)=u+\lambda f(u)$ satisfies Hypothesis H4.

Theorem 2. Assume that $f$ satisfies hypotheses (H1)-(H4). Then for any measurable subset $M \subset B_{N_{0}}^{n}$ and for $\lambda$ sufficiently large, the equation

$$
\left\{\begin{array}{l}
u \in X_{\infty} \\
A u(x)+\lambda f(u(x))=0, x \in \mathbb{Q}_{p}^{n},
\end{array}\right.
$$

has a unique solution $\widetilde{u}$ satisfying

$$
\alpha^{+} \leq \widetilde{u}(x) \leq 1 \text { for } x \in M \text { and }-1 \leq \widetilde{u}(x) \leq \alpha^{-} \text {for } x \in B_{N_{0}}^{n} \backslash M .
$$

Proof. We define

$Y:=\left\{u \in X_{\infty} ; u(x) \in\left[\alpha^{+}, 1\right]\right.$ for $x \in M$ and $u(x) \in\left[-1, \alpha^{-}\right]$for $\left.x \in B_{N_{0}}^{n} \backslash M\right\}$ and the mapping $T: Y \rightarrow X_{\infty}$ as

$$
\begin{aligned}
T u(x) & =u(x)-h\{A u(x)+\lambda f(u(x))\} \\
& =(1-h) u(x)+h J\left(\|x\|_{p}\right) * u(x)-h \lambda f(u(x))
\end{aligned}
$$

for $h>0$. By Lemma 4, $A: Y \rightarrow X_{\infty}$ is well-defined operator, and $u \rightarrow f(u)$ is also a well-defined mapping from $Y$ into $X_{\infty}$ because $f(u)$ is a continuous function satisfying $\lim _{\|x\|_{p} \rightarrow \infty} f(u(x))=0$, here we use that $f(0)=0$.

We show that for $h$ sufficiently small $T$ is a contraction from $Y$ into itself, and since $Y$ is a Banach space, $T$ has a unique fixed point $\widetilde{u}$, which is the desired solution.

Claim 1. For $h$ sufficiently small, if $u(x) \in\left[-1, \alpha^{-}\right] \cup\left[\alpha^{+}, 1\right]$, then $-1 \leq$ $T u(x) \leq 1$. 
Indeed, since $f^{\prime}(u)$ is non-negative and continuous on $\left[-1, \alpha^{-}\right] \cup\left[\alpha^{+}, 1\right]$, we can pick $h$ sufficiently small to get

$$
h \lambda f^{\prime}(u)<1-h \text { for } u \in\left[-1, \alpha^{-}\right] \cup\left[\alpha^{+}, 1\right] .
$$

Notice that (6.3) holds if $0<h<\frac{1}{\left.1+\lambda \max _{u \in[-1, \alpha-}\right] \cup\left[\alpha^{+}, 1\right]} f^{f^{\prime}(u)}$.

From (6.3) with $u(x) \in\left[\alpha^{+}, 1\right]$, we get $h \lambda \int_{u}^{1} f^{\prime}(u) d u \leq(1-h) \int_{u}^{1} d u$, which implies that $(1-h) u-h \lambda f(u) \leq 1-h$, and thus $T u(x) \leq(1-h)+h J\left(\|x\|_{p}\right) *$ $u(x) \leq 1$, since

$$
\left|J\left(\|x\|_{p}\right) * u(x)\right| \leq\|J\|_{L^{1}}\|u\|_{\infty} \leq 1 .
$$

In the case in which $u(x) \in\left[-1, \alpha^{-}\right]$, we get from (6.3) that $h \lambda \int_{-1}^{u} f^{\prime}(u) d u \leq$ $(1-h) \int_{-1}^{u} d u$, which implies that $(1-h) u-h \lambda f(u) \geq-1+h \geq-1$, and thus $T u(x) \geq-1+h J\left(\|x\|_{p}\right) * u(x) \geq-1$, by using (6.4).

Claim 2. $T: Y \rightarrow Y$.

Case $u(x) \in\left[\alpha^{+}, 1\right]$ for $x \in M$.

By using that $u-h\{u+\lambda f(u)\}$ is increasing on $\left[\alpha^{+}, 1\right]$, we have

$$
\begin{gathered}
T u(x) \geq \alpha^{+}-h \alpha^{+}-h \lambda f\left(\alpha^{+}\right)+h J\left(\|x\|_{p}\right) * u(x) \\
\geq \alpha^{+}+h\left\{\alpha^{+} \int_{M} J\left(\|x-y\|_{p}\right) d^{n} y-\alpha^{+}-\lambda f\left(\alpha^{+}\right)\right\} \\
\geq \alpha^{+}+h\left\{\alpha^{+} \int_{M} J\left(\|x-y\|_{p}\right) d^{n} y-\alpha^{+}-\lambda f\left(\alpha^{+}\right)-\int_{\mathbb{Q}_{p}^{n} \backslash M} J\left(\|x-y\|_{p}\right) d^{n} y\right\} \\
=\alpha^{+}-h\left\{\left(1+\alpha^{+}\right) \int_{\mathbb{Q}_{p}^{n} \backslash M} J\left(\|x-y\|_{p}\right) d^{n} y+\lambda f\left(\alpha^{+}\right)\right\} \geq \\
\alpha^{+}-h\left\{\left(1+\alpha^{+}\right)+\lambda f\left(\alpha^{+}\right)\right\},
\end{gathered}
$$

since $\int_{\mathbb{Q}_{p}^{n} \backslash M} J\left(\|x-y\|_{p}\right) d^{n} y \leq 1$. Now, by Condition C6 and Claim 1, $\alpha^{+} \leq$ $T u(x) \leq 1$ for $x \in M$.

Case $u(x) \in\left[-1, \alpha^{-}\right]$for $x \in B_{N_{0}}^{n} \backslash M$.

By using that $u-h\{u+\lambda f(u)\}$ is increasing on $\left[-1, \alpha^{-}\right]$, cf. (6.3),

$$
\begin{gathered}
T u(x) \leq \alpha^{-}-h \alpha^{-}-h \lambda f\left(\alpha^{-}\right)+h J\left(\|x\|_{p}\right) * 1_{B_{N_{0}}^{n} \backslash M}(x) u(x) \\
\leq \alpha^{-}-h\left\{\alpha^{-}-\alpha^{-} \int_{B_{N_{0}}^{n} \backslash M} J\left(\|x-y\|_{p}\right) d^{n} y+\lambda f\left(\alpha^{-}\right)\right\} \\
\leq \alpha^{-}-h\left\{\alpha^{-}+\lambda f\left(\alpha^{-}\right)\right\},
\end{gathered}
$$

since $h \alpha^{-} \int_{B_{N_{0}}^{n} \backslash M} J\left(\|x-y\|_{p}\right) d^{n} y \leq 0$. Now by using Condition C7 and Claim $1,-1 \leq T u(x) \leq \alpha^{-}$for $x \in B_{N_{0}}^{n} \backslash M$.

Claim 3. $T: Y \rightarrow Y$ is a contraction for $h$ sufficiently small. 
Take $u, v$ in $X$, then

$$
\begin{aligned}
\|T u-T v\|_{\infty} & =\|(1-h)(u-v)-h \lambda\{f(u)-f(v)\}+h J *(u-v)\|_{\infty} \\
& =\left\|\left(1-h-h \lambda f^{\prime}(w)\right)(u-v)+h J *(u-v)\right\|_{\infty} \\
& \leq\left|1-h-h \lambda f^{\prime}(w)\right|\|u-v\|_{\infty}+h\|u-v\|_{\infty},
\end{aligned}
$$

with $w=a u+(1-a) v$ for some $0<a<1$. By (6.3), $1-h-h \lambda f^{\prime}(w)>0$ for $h$ sufficiently small, and $1-h-h \lambda f^{\prime}(w) \leq 1$ because $-h\left(1+\lambda f^{\prime}(w)\right) \leq 0$ given that $\lambda f^{\prime}(w) \geq \lambda \delta>0$, cf. Condition C5. Hence

$$
\|T u-T v\|_{\infty} \leq\left(1-h-h \lambda f^{\prime}(w)\right)\|u-v\|_{\infty} \leq\|u-v\|_{\infty} .
$$

By using the fact that $Y$ is a Banach space we conclude that the equation (6.1) has a unique solution $\widetilde{u}$ satisfying (6.2). Now, from Hypothesis $\mathrm{H} 4$ and using that $\widetilde{u}=g_{i}^{-1}(J * \widetilde{u}), i=1,2$, where $g_{i}^{-1}$ is one of the extreme branches of $g^{-1}$, and the fact that $J * \widetilde{u}$ is a continuous function (by the Dominated Convergence Theorem), we conclude that $\widetilde{u}$ is a continuous function.

Remark 5. In Theorem Q ball $B_{N_{0}}^{n}$ can be replaced by a compact subset, however, for the sake of simplicity we use a ball centered at the origin.

Theorem 3. Fix $N_{0} \geq 1$ and assume that supp $J\left(\|x\|_{p}\right) \subset B_{N_{0}}^{n}$, and that $f$ satisfies hypotheses (H1)-(H4).Then for any open and compact subset $M$ contained in $B_{N_{0}}^{n}$ and for $\lambda$ sufficiently large, the equation

$$
\left\{\begin{array}{l}
u \in X_{N_{0}} \\
A_{N_{0}} u(x)+\lambda f(u(x))=0, x \in B_{N_{0}}^{n},
\end{array}\right.
$$

has a unique solution $\widetilde{u}$ satisfying

$$
\alpha^{+} \leq \widetilde{u}(x) \leq 1 \text { for } x \in M \text { and }-1 \leq \widetilde{u}(x) \leq \alpha^{-} \text {for } x \in B_{N_{0}}^{n} \backslash M .
$$

Proof. Due to the fact that $X_{N_{0}}$ is a subspace of $X_{\infty}$ the proof of this result is completely similar to the one given for Theorem 2 . We define

$Y_{N_{0}}:=\left\{u \in X_{N_{0}} ; u(x) \in\left[\alpha^{+}, 1\right]\right.$ for $x \in M$ and $u(x) \in\left[-1, \alpha^{-}\right]$for $\left.x \in B_{N_{0}}^{n} \backslash M\right\}$ and the mapping $T_{N_{0}}: Y_{N_{0}} \rightarrow X_{N_{0}}$ as

$$
\begin{aligned}
T_{N_{0}} u(x) & =u(x)-h\left\{A_{N_{0}} u(x)+\lambda f(u(x))\right\} \\
& =(1-h) u(x)+h J\left(\|x\|_{p}\right) * u(x)-h \lambda f(u(x)) .
\end{aligned}
$$

for $h>0$. By Lemma $3\left(\|x\|_{p}\right) * u(x)$ gives rise to a linear operator from $X_{N}$ into itself, and since

$$
f(u(x))=\sum_{i \in G_{N_{0}}^{n}} f(u(\boldsymbol{i})) \Omega\left(p^{N_{0}}\|x-\boldsymbol{i}\|_{p}\right),
$$

we have $T_{N_{0}} u \in X_{N_{0}}$ if $u \in Y_{N_{0}}$. Now the proof continues as in the proof of Theorem 2

Remark 6. By using the fact that $M$ is open compact, there exists $I_{M}$ a subset of $G_{N_{0}}^{n}$ such that $M=\bigsqcup_{i \in I_{M}} \boldsymbol{i}+\left(p^{N_{0}} \mathbb{Z}_{p}\right)^{n}$. By identifying $u(x)$ with the column vector $[u(i)]_{i \in G_{N_{0}}^{n}}$ and using (6.7), the equation (6.5) takes the form

$$
A^{\left(N_{0}\right)}[u(\boldsymbol{i})]_{\boldsymbol{i} \in G_{N_{0}}^{n}}-\lambda[f(u(\boldsymbol{i}))]_{\boldsymbol{i} \in G_{N_{0}}^{n}}=0,
$$


then, Theorem 3 asserts that the equation (6.8) has a unique solution $\left[\widetilde{u}_{\boldsymbol{i}}\right]_{\boldsymbol{i} \in G_{N_{0}}^{n}}$ satisfying

$$
\alpha^{+} \leq \widetilde{u}(\boldsymbol{i}) \leq 1 \text { for } \boldsymbol{i} \in I_{M} \text { and }-1 \leq \widetilde{u}(\boldsymbol{i}) \leq \alpha^{-} \text {for } \boldsymbol{i} \in G_{N_{0}}^{n} \backslash I_{M}
$$

The proofs of Theorems 2, 3use the same technique as the proof of [12, Theorem 2.1]. However, the hypotheses that we use are simpler than in [12, Theorem 2.1].

\section{A Comparison Theorem}

We consider $\mathbb{Q}_{p}^{n} \times \mathbb{R}$ as a topological space with the product topology. For $T>0$, we set $\mathbb{D}=B_{L}^{n} \times(0, T)$. Notice that the topological closure of $\mathbb{D}$ is $\overline{\mathbb{D}}=B_{L}^{n} \times[0, T]$ and that the boundary of $\mathbb{D}$ is $\mathrm{BdD}=B_{L}^{n} \times\{0\} \cup B_{L}^{n} \times\{T\}$.

Theorem 4. Let $u(x, t), v(x, t): \mathbb{Q}_{p}^{n} \times \mathbb{R} \rightarrow \mathbb{R}$ be functions such that the following conditions hold for $L \geq L_{0}(T)$ for some $L_{0}(T) \in \mathbb{N}$ :

(C1) $u(x, t), v(x, t)$ are continuous functions of $x$ in $B_{L}^{n}$ and continuous differentiable functions of $t$ on $[0, T]$;

(C2) with the notation $P w(x, t):=\frac{\partial w(x, t)}{\partial t}-\left\{J\left(\|x\|_{p}\right) * w(x, t)-w(x, t)\right\}$,

$$
P u(x, t)+\lambda f(u(x, t)) \geq P v(x, t)+\lambda f(v(x, t)) \text { for }(x, t) \in \mathbb{D} ;
$$

(C3) $u(x, 0) \geq v(x, 0)$ for $x$ in $B_{L}^{n}$.

Then under the hypotheses (C1)-(C3), it verifies that

$$
u(x, t) \geq v(x, t) \text { for }(x, t) \in \overline{\mathbb{D}} \text {. }
$$

In addition, if $\|u(\cdot, t)-v(\cdot, t)\|_{\infty} \leq C<\infty$, where $C$ is a constant independent of $t$, there exists $L_{0} \in \mathbb{N}$ such that for $L \geq L_{0}$,

$$
u(x, t) \geq v(x, t) \text { for }(x, t) \in B_{L}^{n} \times[0, \infty) .
$$

Proof. Set $w(x, t):=u(x, t)-v(x, t)$. Then $w(x, 0) \geq 0$ for $x$ in $B_{L}^{n}$ and

$$
P w(x, t)+\lambda f^{\prime}\left(w_{0}\right) w(x, t) \geq 0 \text { for }(x, t) \in \mathbb{D},
$$

where $w_{0}=a u+(1-a) v$, for some $a:=a(x, t)$ satisfying $0<a<1$. By contradiction, assume that $w<0$ at some point of $\overline{\mathbb{D}}$. Set $z(x, t)=e^{-k t} w(x, t)$ with $k$ a positive constant satisfying

$$
\lambda f^{\prime}\left(w_{0}\right)+k>0 \text { in } \overline{\mathbb{D}} .
$$

Then

$$
P w+\lambda f^{\prime}\left(w_{0}\right) w=e^{k t}\left\{P z+z\left(\lambda f^{\prime}\left(w_{0}\right)+k\right)\right\} \geq 0 \text { in } \mathbb{D},
$$

and $z(x, 0)=w(x, 0) \geq 0$ in $B_{L}^{n}$. Since $z$ is negative at some point of $\overline{\mathbb{D}}$,

$$
m:=\min _{(x, t) \in \overline{\mathbb{D}}} z(x, t)<0 \text { and } m=z\left(x_{0}, t_{0}\right) \text { for some }\left(x_{0}, t_{0}\right) \in \overline{\mathbb{D}} .
$$

Three cases occur: (i) $\left(x_{0}, t_{0}\right) \in B_{L}^{n} \times\{0\}$, (ii) $\left(x_{0}, t_{0}\right) \in B_{L}^{n} \times\{T\}$, (iii) $\left(x_{0}, t_{0}\right) \in \mathbb{D}$. The first case is not possible due to condition (C3). We now consider case (iii). By (7.1) we get

$$
P z\left(x_{0}, t_{0}\right) \geq-\left(\lambda f^{\prime}\left(w_{0}\right)+k\right) z\left(x_{0}, t_{0}\right)
$$

and since $\left(\lambda f^{\prime}\left(w_{0}\right)+k\right)>0$ in $\mathbb{D}$, we get $P z\left(x_{0}, t_{0}\right)>0$. A contradiction is derived from Claim 4, and thus this case is not possible.

Claim 4. If $\left(x_{0}, t_{0}\right) \in \mathbb{D}$, then $P z\left(x_{0}, t_{0}\right) \leq 0$ for $L$ sufficiently large. 
The proof requires to consider two subcases: (i) supp $J \subset B_{L}^{n}$ for some $L \in \mathbb{N}$, (ii) supp $J \nsubseteq B_{L}^{n}$ for any $L \in \mathbb{N}$. In the first subcase, $\frac{\partial z\left(x_{0}, t_{0}\right)}{\partial t} \leq 0$ since $z\left(x_{0}, t\right)$ has a global minimum in $[0, T]$, and since

$$
(J * z-z)\left(x_{0}, t_{0}\right)=\int_{B_{L}^{n}} J\left(\left\|x_{0}-y\right\|_{p}\right)\left\{z\left(y, t_{0}\right)-z\left(x_{0}, t_{0}\right)\right\} d^{n} y \geq 0,
$$

hence $P z\left(x_{0}, t_{0}\right) \leq 0$. In the second subcase, $\frac{\partial z\left(x_{0}, t_{0}\right)}{\partial t} \leq 0$ and

$$
\begin{gathered}
(J * z-z)\left(x_{0}, t_{0}\right)=\int_{\mathbb{Q}_{p}^{n}} J\left(\left\|x_{0}-y\right\|_{p}\right)\left\{z\left(y, t_{0}\right)-z\left(x_{0}, t_{0}\right)\right\} d^{n} y \\
=\int_{B_{L}^{n}} J\left(\left\|x_{0}-y\right\|_{p}\right)\left\{z\left(y, t_{0}\right)-z\left(x_{0}, t_{0}\right)\right\} d^{n} y+\int_{\mathbb{Q}_{p}^{n} \backslash B_{L}^{n}} J\left(\left\|x_{0}-y\right\|_{p}\right) z\left(y, t_{0}\right) d^{n} y \\
-z\left(x_{0}, t_{0}\right) \int_{\mathbb{Q}_{p}^{n} \backslash B_{L}^{n}} J\left(\left\|x_{0}-y\right\|_{p}\right) d^{n} y=: I_{0}+I_{1}+I_{2} .
\end{gathered}
$$

Now, $I_{0} \geq 0$ since $z\left(y, t_{0}\right) \geq z\left(x_{0}, t_{0}\right)$ for $y \in B_{L}^{n}$, and $I_{2}>0$ since

$$
\int_{\|y\|_{p}>p^{L}} J\left(\left\|x_{0}-y\right\|_{p}\right) d^{n} y=\int_{\|y\|_{p}>p^{L}} J\left(\|y\|_{p}\right) d^{n} y \text { because } x_{0} \in B_{L}^{n},
$$

this last integral is strictly positive due to the fact that $\operatorname{supp} J \nsubseteq B_{L}^{n}$ for any $L \in \mathbb{N}$, and that $J$ is a continuous function. On the other hand,

$$
\left|I_{1}\right| \leq\left\|z\left(\cdot, t_{0}\right)\right\|_{\infty} \int_{\mathbb{Q}_{p}^{n} \backslash B_{L}^{n}} J\left(\left\|x_{0}-y\right\|_{p}\right) d^{n} y \leq C(T) \int_{\|y\|_{p}>p^{L}} J\left(\|y\|_{p}\right) d^{n} y
$$

since $J\left(\|y\|_{p}\right) \in L^{1}$, for $L$ sufficiently large, $\left|I_{1}\right|<I_{2}$ and therefore $P z\left(x_{0}, t_{0}\right)<0$ for $L \geq L_{0}(T)$ for some $L_{0}(T) \in \mathbb{N}$.

Finally, we consider the case (ii). In this case, since $P z+z\left(\lambda f^{\prime}\left(w_{0}\right)+k\right) \geq 0$ in $\mathbb{D}$, by taking the limit as $(x, t) \rightarrow\left(x_{0}, T\right)$ we get $P z\left(x_{0}, T\right) \geq 0$. The argument given for Claim 4 also works in the case $\left(x_{0}, T\right)$ because $z\left(x_{0}, t\right)$ is differentiable at $t=T$. Therefore case (ii) is also impossible.

Lemma 9. Set $\bar{u}(x, t):=\widetilde{u}(x)+\epsilon e^{-\beta t}, \underline{u}(x, t):=\widetilde{u}(x)-\epsilon e^{-\beta t}$, where $\widetilde{u}(x)$ is the function given in Theorem $\mathbb{2}, \epsilon, \beta>0$, and $x \in \mathbb{Q}_{p}^{n}, t \geq 0$. If $\epsilon, \beta$ are sufficiently small, then

$$
P \bar{u}(x, t)+\lambda f(\bar{u}(x, t)) \geq 0 \text { for } x \in \mathbb{Q}_{p}^{n}, t \geq 0
$$

and

$$
P \underline{u}(x, t)+\lambda f(\underline{u}(x, t)) \leq 0 \text { for } x \in \mathbb{Q}_{p}^{n}, t \geq 0 .
$$

Proof. We show (7.2), the other inequality is established in the same way. We first note that

$$
P \bar{u}(x, t)+\lambda f(\bar{u}(x, t))=-\beta \epsilon e^{-\beta t}+\lambda\left\{f\left(\widetilde{u}(x)+\epsilon e^{-\beta t}\right)-f(\widetilde{u}(x))\right\} .
$$

By using that $\widetilde{u}(x)$ is bounded and the Taylor Expansion Theorem with $f \in C^{2}(\mathbb{R})$,

$$
\left|f\left(\widetilde{u}(x)+\epsilon e^{-\beta t}\right)-f(\widetilde{u}(x))-\epsilon e^{-\beta t} f^{\prime}(\widetilde{u}(x))\right| \leq C \epsilon^{2} e^{-2 \beta t},
$$


for some $C>0$. Finally, by (H4) and by choosing $\epsilon, \beta$ sufficiently small,

$$
f\left(\widetilde{u}(x)+\epsilon e^{-\beta t}\right) \geq f(\widetilde{u}(x))+\epsilon e^{-\beta t} f^{\prime}(\widetilde{u}(x))-C \epsilon^{2} e^{-2 \beta t} \geq 0 .
$$

Remark 7. If in Lemma 9, operator Pw $(x, t)$ is replaced by

$$
\frac{\partial w(x, t)}{\partial t}-\left\{J_{N}\left(\|x\|_{p}\right) * w(x, t)-j_{N} w(x, t)\right\}
$$

and take $x \in B_{L}^{n}, t \geq 0$, with $L$ sufficiently large, then (7.2)-7.3) are valid for $x \in B_{L}^{n}, t \geq 0$.

Let us consider the following Cauchy problem:

$$
\left\{\begin{array}{l}
\frac{\partial u(x, t)}{\partial t}=J\left(\|x\|_{p} * u(x, t)\right)-u(x, t)-\lambda f(u(x, t)), \\
u(x, 0)=u_{0}(x),
\end{array}\right.
$$

where $(x, t) \in \mathbb{Q}_{p}^{n} \times[0, \infty)$, and $u(x, t): \mathbb{Q}_{p}^{n} \times[0, \infty) \rightarrow \mathbb{R}$ is a bounded continuous function in $x$ for any fixed $t \geq 0$, which is continuously differentiable in $t \in[0, \infty)$ for any fixed $x \in \mathbb{Q}_{p}^{n}$.

Corollary 1. Assume that $u(x, t)$ is a solution of Cauchy problem (7.4) with $u_{0}(x)$ a bounded function satisfying $\widetilde{u}(x)-\epsilon \leq u_{0}(x) \leq \widetilde{u}(x)+\epsilon$, with $\epsilon$ sufficiently small so that Lemma 9 holds. Then $\underline{u}(x, t) \leq u(x, t) \leq \bar{u}(x, t)$ for $(x, t) \in \mathbb{Q}_{p}^{n} \times[0, \infty)$.

Proof. The result follows from Theorem 4 and Lemma 9.

Lest us consider the following Cauchy problem:

$$
\left\{\begin{array}{l}
\frac{\partial u(x, t)}{\partial t}=J_{N}\left(\|x\|_{p} * u(x, t)\right)-u(x, t)-\lambda f(u(x, t)) \\
u(x, 0)=u_{0}(x)
\end{array}\right.
$$

where $(x, t) \in B_{N}^{n} \times[0, \infty)$, and $u(x, t): B_{N}^{n} \times[0, \infty) \rightarrow \mathbb{R}$ is a continuous function in $x$ for any fixed $t \geq 0$, which is continuously differentiable in $t \in[0, \infty)$ for any fixed $x \in B_{N}^{n}$.

Corollary 2. Assume that $N$ is sufficiently large and that $u(x, t)$ is a solution of Cauchy problem (7.5) with $u_{0}(x) \in X_{N}$ satisfying $\widetilde{u}(x)-\epsilon \leq u_{0}(x) \leq \widetilde{u}(x)+\epsilon$, with $\epsilon$ sufficiently small so that Lemma 9 and Remark 7 hold. Then $\underline{u}(x, t) \leq$ $u(x, t) \leq \bar{u}(x, t)$ for $(x, t) \in B_{N}^{n} \times[0, \infty)$.

Proof. The result follows from Theorem 4 and Remark 7

\section{The Cauchy Problem}

Theorem 5. Consider the Cauchy problem:

$$
\begin{cases}u(x, t) \in C\left([0, T], X_{\infty}\left(\mathbb{Q}_{p}^{n}\right)\right) \cap C^{1}\left([0, T], X_{\infty}\left(\mathbb{Q}_{p}^{n}\right)\right), & T>0 \\ \frac{\partial u(x, t)}{\partial t}=J\left(\|x\|_{p}\right) * u(x, t)-u(x, t)-\lambda f(u(x, t)), & t \in[0, T] \\ u(x, 0)=u_{0}(x), & \end{cases}
$$

with $u_{0}(x) \in X_{\infty}\left(\mathbb{Q}_{p}^{n}\right)$ satisfying $\widetilde{u}(x)-\epsilon \leq u_{0}(x) \leq \widetilde{u}(x)+\epsilon$, with $\lambda, \widetilde{u}(x)$ as in Theorem 1 , and with $\epsilon$ sufficiently small so that Lemma 9 holds. Then, the initial value problem (8.1) has a unique solution satisfying $\underline{u}(x, t) \leq u(x, t) \leq \bar{u}(x, t)$ for $(x, t) \in \mathbb{Q}_{p}^{n} \times[0, \infty)$. In addition, $u(x, t)$ satisfies then $\lim _{t \rightarrow \infty}\|u(x, t)-\widetilde{u}(x)\|_{\infty}=$ 0 . 
Proof. We recall that $-A u(x, t)=J\left(\|x\|_{p}\right) * u(x, t)-u(x, t)$. By Lemma 4, $A$ gives rise to a linear bounded operator from $X_{\infty}$ onto itself. On the other hand, $A$ is $m$-dissipative, i.e. there exists $\nu_{0}>0$ such that for all $h \in X_{\infty}$ there exists a solution $u \in X_{\infty}$ of $u-\nu_{0} A u=h$, cf. [16, Proposition 2.2.6]. Indeed, consider the operator

$$
\begin{aligned}
T: X_{\infty} & \rightarrow X_{\infty} \\
u & \rightarrow-\nu J * u+\nu u+h,
\end{aligned}
$$

with $\nu>0$. By Lemma 4, $T$ is well-defined and $|T u-T v| \leq 2 \nu\|u-v\|_{\infty}$, thus if $0<2 \nu<1, T$ is a contraction and by the Banach Fixed Point Theorem there exits a unique $u \in X_{\infty}$ such that $T u=u$, which implies that $A$ is an $m$-dissipative operator on $X_{\infty}$. By the Hille-Yosida-Phillips Theorem, $-A$ is the generator of a contraction semigroup $e^{-t A}$ on $X_{\infty}$, see e.g. [16, Theorem 3.4.4]. Now, any solution of (8.1) is a solution of the following integral equation:

$$
u(x, t)=e^{-t A} u_{0}(x)+\int_{0}^{t} e^{-(t-s) A} f(u(x, s)) d s \text { for } t \in[0, T], T>0,
$$

cf. [16, Lemma 4.1.1]. By using that $\left\|u_{0}(x)\right\|_{\infty} \leq\|\widetilde{u}(x)\|_{\infty}+\epsilon \leq 1+\epsilon=: M<\infty$, there exists a unique solution $u(x, t) \in C\left(\left[0, T_{M}\right], X_{\infty}\left(\mathbb{Q}_{p}^{n}\right)\right)$ of [8.2), cf. [16, Proposition 4.3.3]. Then, two cases occur: (i) $T_{M}=\infty$, i.e. there exists a global solution for (8.1); (ii) $T_{M}<\infty$ and $\lim _{t \rightarrow T_{M}}\|u(\cdot, t)\|_{\infty}=\infty$, cf. [16, Theorem 4.3.4], now by Corollary 1, $\|u(\cdot, t)\|_{\infty} \leq M$, therefore $T_{M}=\infty$. Finally, by using Corollary 1 and Lemma 9, $\lim _{t \rightarrow \infty}\|u(x, t)-\widetilde{u}(x)\|_{\infty}=0$.

By using the same reasoning we obtain the following finite dimensional version of Theorem 5 .

Theorem 6. Consider the Cauchy problem:

$$
\begin{cases}u(x, t) \in C\left([0, T], X_{N}\right) \cap C^{1}\left([0, T], X_{N}\right), & T>0 \\ \frac{\partial u(x, t)}{\partial t}=J_{N}\left(\|x\|_{p}\right) * u(x, t)-u(x, t)-\lambda f(u(x, t)), & x \in B_{N}^{n}, t \in[0, T] \\ u(x, 0)=u_{0}(x), & \end{cases}
$$

with $N$ sufficiently large, $u_{0}(x) \in X_{N}$ satisfying $\widetilde{u}(x)-\epsilon \leq u_{0}(x) \leq \widetilde{u}(x)+\epsilon$, with $\lambda, \widetilde{u}(x)$ as in Theorem [3, and with $\epsilon$ sufficiently small so that Lemma 9 and Remark 7 hold. Then the initial value problem (8.3) has a unique solution satisfying $\underline{u}(x, t) \leq u(x, t) \leq \bar{u}(x, t)$ for $(x, t) \in B_{N}^{n} \times[0, \infty)$. In addition, $u(x, t)$ satisfies $\lim _{t \rightarrow \infty}\|u(x, t)-\widetilde{u}(x)\|_{\infty}=0$.

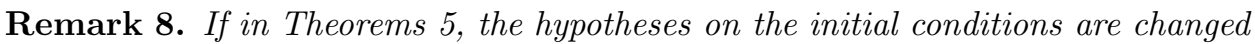
to "with $u_{0}(x) \in X_{\infty}\left(\mathbb{Q}_{p}^{n}\right)$ satisfying $-1 \leq u_{0}(x) \leq 1$," then there exists a unique solution satisfying $-1 \leq u(x, t) \leq 1$ for $x \in \mathbb{Q}_{p}^{n}$ and $t \geq 0$. A similar result is obtained if in Theorem 6 , the hypotheses on the initial conditions are changed to "with $N$ sufficiently large, $u_{0}(x) \in X_{N}$ satisfying $-1 \leq u_{0}(x) \leq 1$."

\section{Finite Approximations}

In this section we study finite approximations to the solutions of

$$
\left\{\begin{array}{l}
\frac{\partial u(x, t)}{\partial t}+A u(x, t)=-\lambda f(u(x, t)), \quad x \in \mathbb{Q}_{p}^{n}, \quad t \geq 0 \\
u(x, 0)=u_{0}(x),
\end{array}\right.
$$

where function $f(u)$ satisfies all the conditions given in Section 6 . 
Lemma 10 (Condition B). For $N \geq 1, A_{N} \in \mathfrak{B}\left(X_{N}, X_{N}\right)$, in addition,

$$
\left\|e^{-A_{N} t}\right\| \leq 1 \text { for } t \geq 0, N \geq 1 \text {. }
$$

Proof. We recall that $e^{-t A_{N}}=e^{-t A^{(N)}}$ for $t \geq 0$, since $e^{-t A^{(N)}}=e^{-t S A^{(N)} S^{-1}}$ for any invertible matrix $S$ and $t \geq 0$. Hence

$$
\left\|e^{-t A_{N}}\right\|=\left\|e^{-t A^{(N)}}\right\| \leq 1 \text { for } t \geq 0
$$

because by Theorem 11 (i), $P^{(N)}(t)=e^{-t A^{(N)}}$ satisfies $P^{(N)}(t) \mathbf{1}=\mathbf{1}$ for $t \geq 0$, where 1 the unit vector which is a vector having all its entries equal to one. With $P^{(N)}(t)=\left[P_{\mathbf{i} j}^{(N)}(t)\right]$, with $P_{\mathbf{i} j}^{(N)}(t) \geq 0$, and taking $\varphi \in X_{N}$ satisfying $\|\varphi\|_{\infty}=1$, we have

$$
\left\|P^{(N)}(t) \varphi\right\|_{\infty}=\max _{\boldsymbol{i}}\left|\sum_{\boldsymbol{j}} P_{\boldsymbol{i} \boldsymbol{j}}^{(N)}(t) \varphi(\boldsymbol{j})\right| \leq \max _{\boldsymbol{i}} \sum_{\boldsymbol{j}} P_{\boldsymbol{i} \boldsymbol{j}}^{(N)}(t)=1
$$

for $t \geq 0$.

Remark 9. $A$ is a linear bounded operator on $X_{\infty}$, and since $\mathcal{D}\left(\mathbb{Q}_{p}^{n}\right)$ is dense in $X_{\infty}, A$ is completely determined by its restriction to $\mathcal{D}\left(\mathbb{Q}_{p}^{n}\right)$. For an easy crossreferencing with [29, we say 'A is densely defined linear operator in $X_{\infty}$ '.

Lemma 11 (Condition C'). A is densely defined linear operator in $X_{\infty}$, there exists $\lambda_{0} \in(-\infty, 0) \cap \rho(A)$ and

$$
\lim _{N \rightarrow \infty}\left\|A_{N} P_{N} \varphi-P_{N} A \varphi\right\|_{\infty}=\lim _{N \rightarrow \infty}\left\|E_{N} P_{N} \varphi-\varphi\right\|_{\infty}=0 \text { for all } \varphi \in \mathcal{D}\left(\mathbb{Q}_{p}^{n}\right) .
$$

Proof. The existence of $\lambda_{0}$ follow from Lemma 4. Take $\varphi \in \mathcal{D}\left(\mathbb{Q}_{p}^{n}\right) \subset X_{\infty}\left(\mathbb{Q}_{p}^{n}\right)$, then $\varphi \in \mathcal{D}_{N_{0}}^{-N_{0}} \subset \mathcal{D}_{N}^{-N}$, for some $N_{0} \geq 1$ and for every $N \geq N_{0}$, in addition, $A \varphi=A_{N_{0}} \varphi$. Indeed,

$$
\begin{gathered}
A \varphi=-\int_{\mathbb{Q}_{p}^{n}} J\left(\|x-y\|_{p}\right)\{\varphi(y)-\varphi(x)\} d^{n} y \\
=-\int_{\mathbb{Q}_{p}^{n}} J\left(\|x-y\|_{p}\right)\left\{\Omega\left(p^{N_{0}}\|y\|_{p}\right) \varphi(y)-\Omega\left(p^{N_{0}}\|p\|_{p}\right) \varphi(x)\right\} d^{n} y \\
=-\int_{B_{N_{0}}^{n}} \Omega\left(p^{N_{0}}\|x-y\|_{p}\right) J\left(\|x-y\|_{p}\right)\left\{\Omega\left(p^{N_{0}}\|y\|_{p}\right) \varphi(y)\right. \\
\left.-\Omega\left(p^{N_{0}}\|p\|_{p}\right) \varphi(x)\right\} d^{n} y=-\int_{B_{N_{0}}^{n}} J_{N_{0}}\left(\|x-y\|_{p}\right)\{\varphi(y)-\varphi(x)\} d^{n} y=A_{N_{0}} \varphi .
\end{gathered}
$$

Now, since $\left.P_{N}\right|_{X_{N_{0}}}=P_{N_{0}}$ for $N \geq N_{0}, P_{N} A \varphi=P_{N}\left(A_{N_{0}} \varphi\right)=P_{N_{0}}\left(A_{N_{0}} \varphi\right)=$ $A_{N_{0}} \varphi=A \varphi$, and since $\left.A_{N}\right|_{X_{N_{0}}}=A_{N_{0}}$ for $N \geq N_{0}, A_{N} P_{N} \varphi=A_{N}\left(P_{N_{0}} \varphi\right)=$ $A_{N_{0}}\left(P_{N_{0}} \varphi\right)=A_{N_{0}} \varphi=A \varphi$, therefore $\left\|A_{N} P_{N} \varphi-P_{N} A \varphi\right\|_{\infty}=0$ for $N \geq N_{0}$. On the other hand, since $\left.E_{N}\right|_{X_{N_{0}}}=E_{N_{0}}$ for $N \geq N_{0}, E_{N} P_{N} \varphi=E_{N}\left(P_{N_{0}} \varphi\right)=$ $E_{N_{0}}\left(P_{N_{0}} \varphi\right)=E_{N_{0}} \varphi=\varphi$, which implies that $\left\|E_{N} P_{N} \varphi-\varphi\right\|_{\infty}=0$ for $N \geq N_{0}$. 
Corollary 3 (Condition C). Assume Conditions $A$ and $B$, then $A$ is densely defined linear operator in $X_{\infty}$, and there exists $\lambda_{0} \in(-\infty, 0) \cap \rho(A)$ such that for all $\varphi$ in a dense subset of $X_{\infty}$,

$$
\lim _{N \rightarrow \infty}\left\|E_{N}\left(A_{N}-\lambda_{0}\right)^{-1} P_{N} \varphi-\left(A-\lambda_{0}\right)^{-1} \varphi\right\|_{\infty}=0 .
$$

Proof. See e.g. Lemma 5.4.1 in [29].

9.1. Finite Approximations for $p$-adic reaction-ultradiffusion equations. Our goal is to approximate the solution $u(x, t)$ of the Cauchy Problem (9.1) in $X_{\infty}$ using only that $u_{0}(x) \in X_{\infty}$ and $-1 \leq u_{0}(x) \leq 1$. The techniques for constructing such approximations are well-known, here we use reference [29, Section 5.4]. It is possible to approximate $u(x, t)$ without using any a priori information on the initial solution, however this requires to impose to the nonlinearity $f$ to be globally Lipschitz, this last condition reduces significantly the potentials $W$ to which we can apply our results.

The discretization of the Cauchy problem (9.1) in the spaces $X_{N}$ takes the following form:

$$
\left\{\begin{array}{l}
\frac{d}{d t} u_{N}(t)+A_{N} u_{N}(t)=-\lambda P_{N} f\left(E_{N} u_{N}(t)\right) \\
u_{N}(0)=P_{N} u_{0}
\end{array}\right.
$$

By taking $P_{N} u_{0}(x)=\sum_{\boldsymbol{i} \in G_{N}^{n}} u_{0}(\boldsymbol{i}) \Omega\left(p^{N}\|x-\boldsymbol{i}\|_{p}\right)$ and identifying $u_{N}(t)$ with the column vector $\left[u_{N}(\boldsymbol{i}, t)\right]_{\boldsymbol{i} \in G_{N}^{n}}$, we can rewrite the Cauchy problem (9.2) as

$$
\left\{\begin{array}{l}
\frac{d}{d t}\left[u_{N}(\boldsymbol{i}, t)\right]_{\boldsymbol{i} \in G_{N}^{n}}+A^{(N)}\left[u_{N}(\boldsymbol{i}, t)\right]_{I \in G_{N}^{n}}=-\lambda\left[f\left(u_{N}(\boldsymbol{i}, t)\right)\right]_{\boldsymbol{i} \in G_{N}^{n}} \\
{\left[u_{N}(\boldsymbol{i}, 0)\right]_{\boldsymbol{i} \in G_{N}^{n}}=\left[u_{0}(\boldsymbol{i})\right]_{\boldsymbol{i} \in G_{N}^{n}},}
\end{array}\right.
$$

cf. Lemma 6 .

Theorem 7. (i) $-A$ is the generator of a strongly continuous semigroup $\left\{e^{-t A}\right\}_{t \geq 0}$ on $X_{\infty}$. Moreover, $\left\|e^{-t A}\right\| \leq 1$ for $t \geq 0$ and

$$
\lim _{N \rightarrow \infty} \sup _{t \geq 0} e^{b t}\left\|E_{N} e^{-A_{N} t} P_{N} \varphi-e^{-t A} \varphi\right\|_{\infty}=0 \text { for all } \varphi \in X_{\infty}, b \in(0, \infty) .
$$

(ii) Take $u_{0}(x) \in X$ with $-1 \leq u_{0}(x) \leq 1$. Let $u$ be the solution of (9.1) and let $u_{N}$ be the solution of (9.2). Then

$$
\lim _{N \rightarrow \infty} \sup _{0 \leq t \leq T}\left\|E_{N} u_{N}(t)-u(t)\right\|_{\infty}=0 .
$$

Proof. The first part follows from Conditions A, B, C by using Theorem 5.4.2 in [29. The proof of the second part is based on the estimation of the $\|\cdot\|_{\infty}$-norm of the difference of a mild solution of (9.1) and a mild solution of (9.2). The required estimation follows from conditions A, B, C, D by using Theorem 5.4.7 in [29].

Acknowledgement. The author wishes to thank to Sergii Torba, the editors of Nonlinearity, and the referees for many useful comments and discussions, which led to an improvement of this work. In addition, the author thanks Luis Gorostiza for telling him about reference [17. 


\section{REFERENCES}

[1] Albeverio S., Khrennikov A. Yu., Shelkovich V. M., Theory of $p$-adic distributions: linear and nonlinear models. London Mathematical Society Lecture Note Series, 370. Cambridge University Press, Cambridge, 2010.

[2] Alberti Giovanni, Bellettini Giovanni, A non-local anisotropic model for phase transitions: asymptotic behaviour of rescaled energies, European J. Appl. Math. 9 (1998), no. 3, 261-284.

[3] Alberti Giovanni, Bellettini Giovanni, A nonlocal anisotropic model for phase transitions. I. The optimal profile problem, Math. Ann. 310 (1998), no. 3, 527-560.

[4] Andreu-Vaillo Fuensanta, Mazón José M., Rossi Julio D., Toledo-Melero J. Julián, Nonlocal diffusion problems. Mathematical Surveys and Monographs, 165. American Mathematical Society, Providence, RI; Real Sociedad Matemática Española, Madrid, 2010.

[5] Avetisov V. A., Bikulov A. Kh., Zubarev, A. P., First passage time distribution and the number of returns for ultrametric random walks, J. Phys. A 42 (2009), no. 8, 085003, 18 pp.

[6] Avetisov V. A., Bikulov A. Kh., Osipov V. A., p-adic description of characteristic relaxation in complex systems, J. Phys. A 36 (2003), no. 15, 4239-4246.

[7] Avetisov V. A., Bikulov A. H., Kozyrev S. V., Osipov V. A., p-adic models of ultrametric diffusion constrained by hierarchical energy landscapes, J. Phys. A 35 (2002), no. 2, 177-189.

[8] Avetisov V. A., Bikulov A. Kh., Kozyrev S. V., Description of logarithmic relaxation by a model of a hierarchical random walk. (Russian) Dokl. Akad. Nauk 368 (1999), no. 2, 164-167.

[9] Avetisov V. A., Bikulov A. H., Kozyrev S. V.m Application of $p$-adic analysis to models of breaking of replica symmetry, J. Phys. A 32 (1999), no. 50, 8785-8791.

[10] Bachas Constantin P., Huberman B. A., Complexity and ultradiffusion, J. Phys. A 20 (1987), no. 14, 4995-5014.

[11] Bates Peter W., Fife, Paul C., Ren Xiaofeng, Wang Xuefeng, Traveling waves in a convolution model for phase transitions, Arch. Rational Mech. Anal. 138 (1997), no. 2, 105-136.

[12] Bates Peter W., Chmaj Adam, An integrodifferential model for phase transitions: stationary solutions in higher space dimensions, J. Statist. Phys. 95 (1999), no. 5-6, 1119-1139.

[13] Becker O. M., Karplus M., The topology of multidimensional protein energy surfaces: theory and application to peptide structure and kinetics, J. Chem.Phys. 106, 1495-1517 (1997).

[14] Bendikov A., Heat kernels for isotropic-like Markov generators on ultrametric spaces: a survey, p-Adic Numbers Ultrametric Anal. Appl. 10 (2018), no. 1, 1-11.

[15] Bikulov Albert Kh., On solution properties of some types of p-adic kinetic equations of the form reaction-diffusion, p-Adic Numbers Ultrametric Anal. Appl. 2 (2010), no. 3, 187-206.

[16] Cazenave Thierry, Haraux Alain, An introduction to semilinear evolution equations. Oxford University Press, 1998.

[17] Dellacherie Claude, Martinez Servet, San Martin Jaime, Inverse $M$-matrices and ultrametric matrices. Lecture Notes in Mathematics, 2118. Springer, 2014.

[18] Dragovich B., Khrennikov A. Yu., Kozyrev S. V., Volovich, I. V., On p-adic mathematical physics, p-Adic Numbers Ultrametric Anal. Appl. 1 (2009), no. 1, 1-17.

[19] Fife Paul C., Mathematical aspects of reacting and diffusing systems. Lecture Notes in Biomathematics, 28. Springer-Verlag, Berlin-New York, 1979.

[20] Frauenfelder H, Chan S. S., Chan W. S. (eds), The Physics of Proteins. Springer-Verlag, 2010.

[21] Grindrod Peter, Patterns and waves. The theory and applications of reaction-diffusion equations. Oxford Applied Mathematics and Computing Science Series. The Clarendon Press, Oxford University Press, New York, 1991.

[22] Kochubei Anatoly N., Pseudo-differential equations and stochastics over non-Archimedean fields. Marcel Dekker, Inc., New York, 2001.

[23] Khrennikov Andrei, Kozyrev Sergei, Zúñiga-Galindo W. A., Ultrametric Equations and its Applications. Encyclopedia of Mathematics and its Applications (168). Cambridge University Press, 2018.

[24] Kozyrev S. V., Methods and Applications of Ultrametric and p-Adic Analysis: From Wavelet Theory to Biophysics, Sovrem. Probl. Mat., 12, Steklov Math. Inst., RAS, Moscow, 2008, 3-168.

[25] Khrennikov A.Yu., Kozyrev S.V., Replica symmetry breaking related to a general ultrametric space I: replica matrices and functionals, Physica A: Statistical Mechanics and its Applications, V.359 (2006), P.222-240. 
[26] Khrennikov A.Yu., Kozyrev S.V., Replica symmetry breaking related to a general ultrametric space II: RSB solutions and the $\$ \mathrm{n} \backslash$ to $0 \$$ limit, Physica A: Statistical Mechanics and its Applications, V.359 (2006), P.241-266.

[27] Khrennikov A.Yu., Kozyrev S.V., Replica symmetry breaking related to a general ultrametric space III: The case of general measure. Physica A: Statistical Mechanics and its Applications, V.378 (2007), N.2. P.283-298.

[28] Mézard Marc, Parisi Giorgio, Virasoro Miguel Angel, Spin glass theory and beyond. World Scientific, 1987.

[29] Miklavčič Milan, Applied functional analysis and partial differential equations. World Scientific Publishing Co., Inc., River Edge, NJ, 1998.

[30] Ogielski Andrew T., Stein D. L., Dynamics on ultrametric spaces, Phys. Rev. Lett. 55 (1985), no. $15,1634-1637$.

[31] Rammal R., Toulouse G., Virasoro M. A., Ultrametricity for physicists, Rev. Modern Phys. 58 (1986), no. 3, 765-788.

[32] Smoller Joel, Shock waves and reaction-diffusion equations. Springer-Verlag, New York, 1994.

[33] Taibleson M. H., Fourier analysis on local fields. Princeton University Press, 1975.

[34] Torresblanca-Badillo Anselmo, Zúñiga-Galindo W. A., Ultrametric Diffusion, Exponential Landscapes, and the First Passage Time Problem, arXiv:1511.08757

[35] Ueyama Eizo, Hosoe Shigeyuki, Reaction-diffusion equation on a graph and phase transition on bistable media in Proceedinds of SICE Annual Conference, August 20-23, 2012, Akita University, Akita, Japan, pp. 1798-1801.

[36] Vladimirov V. S., Volovich I. V., Zelenov E. I., p-adic analysis and mathematical physics. World Scientific, 1994.

[37] Yin G. George, Zhang Qing, Continuous-time Markov chains and applications. A two-timescale approach. 2nd ed. Springer 2013.

[38] Zúñiga-Galindo W. A. , Pseudodifferential equations over non-Archimedean spaces. Lectures Notes in Mathematics 2174, Springer, 2016.

[39] Zwanzig Robert, Simple model of protein folding kinetics, Proc. Nat. Acad. Sci. U.S.A. 92 (1995), no. 21, 9801-9804.

Centro de Investigación y de Estudios Avanzados del Instituto Politécnico Nacional, Departamento de Matemáticas, Unidad Querétaro, Libramiento Norponiente \#2000, Fracc. Real de Juriquilla. Santiago de Querétaro, Qro. 76230, México.

E-mail address: wazuniga@math.cinvestav.edu.mx 